\title{
Charged-Higgs Collider Signals with or without Flavor
}

\author{
Stefan Dittmaier, ${ }^{1}$ Gudrun Hiller, ${ }^{2}$ Tilman Plehn, ${ }^{3}$ and Michael Spannowsky ${ }^{4}$ \\ ${ }^{1}$ Max-Planck-Institut für Physik (Werner-Heisenberg-Institut), München, Germany \\ ${ }^{2}$ Institut für Physik, Universität Dortmund, Germany \\ ${ }^{3}$ SUPA, School of Physics, University of Edinburgh, Scotland \\ ${ }^{4} A S C$, Department für Physik, Ludwig-Maximilians-Universität München, Germany
}

(Dated: November 2, 2018)

\begin{abstract}
A charged Higgs boson is a clear signal for an extended Higgs sector, as for example predicted by supersymmetry. Squark mixing can significantly change the pattern of charged-Higgs production and most notably circumvent the chiral suppression for single Higgs production. We evaluate the LHC discovery potential in the light of flavor physics, in the single-Higgs production channel and in association with a hard jet for small and moderate values of $\tan \beta$. Thoroughly examining current flavor constraints we find that non-minimal flavor structures can have a sizeable impact, but tend to predict moderate production rates. Nevertheless, charged-Higgs searches will probe flavor structures not accessible to rare kaon, bottom, or charm experiments, and can invalidate the assumption of minimal flavor violation.
\end{abstract}

\section{INTRODUCTION}

Understanding the nature of electroweak symmetry breaking is the most important endeavor in high-energy physics over the coming years. With the LHC close to delivering data and probing a new energy range in particle physics, we expect to be close to solving this one remaining puzzle in the Standard Model.

The Standard Model chooses a particularly simple approach to electroweak symmetry breaking: all masses are created by one Higgs doublet acquiring a vacuum expectation value. This one doublet and its conjugate give mass to up-type and down-type fermions. For example, supersymmetry does not allow for this simple mechanism. We need two Higgs doublets to give mass to all fermions, if we want the Higgs fields to respect supersymmetry and if we want to avoid anomalies arising from fermionic supersymmetric Higgsinos. Such an extended model with each Higgs doublet coupling exclusively to up-type or down-type fermions is generally referred to as a two-Higgs-doublet model of type II [1]. Taking into account electroweak precision data [2], a typical two-Higgs-doublet model will predict a light Higgs scalar and a set of additional heavy Higgs modes. In the most prominent two-Higgs-doublet model (the MSSM Higgs sector) there is no doubt that we will see the light scalar Higgs in the usual Standard Model search channels [3]. Unfortunately, to positively identify an extended Higgs sector it might not be sufficient to simply study this light Higgs [4]. An additional heavy charged Higgs is the most distinct signature of a second Higgs doublet. In contrast to a heavy neutral scalar, it does not get faked by states that are not linked to the Higgs sector.

Over the years, many charged-Higgs search strategies at the LHC have been proposed and studied. For a pure MSSM-type two-Higgs-doublet model the entire leading-order parameter space is described by the chargedHiggs mass $m_{H^{+}}$and $\tan \beta$, where $\tan \beta$ is the ratio of the two vacuum expectation values. Almost all of the LHC search strategies make use of a particularity in the type-II two-Higgs-doublet model: the heavy-quark Yukawas $y_{q}$ to the heavy Higgs states are governed by $y_{b} \tan \beta$ and by $y_{t} / \tan \beta$. The most promising strategy for finding a charged Higgs at the LHC will therefore include coupling it to incoming or outgoing bottoms.

The most promising charged-Higgs production channel is in association with a top quark [5, 6 ,, , , 8]. The rate can consistently be computed in a 5 -flavor or in a 4-flavor scheme, i.e. with or without using bottom parton densities 9]. Because of the complexity of the top-associated final state, a charged-Higgs decay to hadronic $\tau^{+} \nu[10,11]$ is easier to extract from the background than the (likely undetectable) decay to $t \bar{b}$ [12, 13]. Recently, it has been shown that the search for a light charged Higgs in anomalous top decays $t \rightarrow H^{+} b \rightarrow\left(\tau^{+} \nu\right) b$ can be merged nicely with the search for a charged Higgs produced with a top quark $\bar{b} g \rightarrow \bar{t} H^{+} \rightarrow \bar{t}\left(\tau^{+} \nu\right)$ [8, 14].

Unfortunately, all strategies described above fail for small $\tan \beta$. The bottom-induced search channels only cover $\tan \beta \gtrsim 20$, leaving a hole $\tan \beta=2 \cdots 20$ in the parameter space. In the MSSM in this region we might only see a light SM-like Higgs, unless we are lucky enough to produce light Higgses in pairs coming from a resonant heavy neutral Higgs [15]. There are several ideas how to cover this region searching for a charged Higgs, such as, e.g., the production in association with a $W$ [16] or pair production. The latter occurs at tree level with incoming bottom quarks, $b \bar{b} \rightarrow H^{+} H^{-}$, it can also be loop mediated, $g g \rightarrow H^{+} H^{-}$, or for low and intermediate $\tan \beta$ we can search for $q \bar{q} \rightarrow H^{+} H^{-}$[17]. Unfortunately, none of these strategies are too promising, because the rates without $\tan \beta$ enhancement are small. 
Looking beyond bottom-mediated production channels reveals an opportunity linked to charged-Higgs searches: while it is well known how to absorb the leading supersymmetric loops into an effective bottom Yukawa coupling [8, 18], the production via light-flavor quarks can be heavily affected by the flavor structure of the model embedding the two Higgs doublets. Within the Standard Model flavor symmetry breaking is governed solely by the Yukawa interactions. This simple, highly predictive mechanism is successful in explaining a multitude of flavor-changing quark transitions. Applying this concept to extensions of the Standard Model leads to the notion of minimal flavor violation (MFV) [19]: in an MFV model there are no other sources of flavor violation other than the Yukawas, the spurions of flavor symmetry breaking. For the case of the MSSM with unbroken $R$ parity, the MFV condition is automatically satisfied for supersymmetric gauge couplings $(D$ terms) and for scalar couplings derived from the superpotential ( $F$ terms). However, general soft SUSY breaking introduces new sources of flavor violation. In MFV (i) all soft scalar squark masses need to be diagonal in flavor space and (ii) all triscalar $A$-terms describing the squark-squark-Higgs couplings have to be proportional to the Yukawas. Corrections consistent with the Standard Model flavor symmetry are induced by higher powers in the Yukawas [19, 20, 21]. This set of MFV assumptions automatically passes a large fraction of experimental constraints.

Such an MFV assumption is not necessary. While some flavor-non-diagonal MSSM couplings are tightly constrained, others can be of order one (see e.g. 22, 23, 24]). In general, constraints from flavor-changing-neutralcurrent (FCNC) $K$ - and $B$-physics data having external down-type quarks are stronger on flavor violation among down-squarks, because down-squark effects can occur via strongly interacting gluino loops, as opposed to upsquark effects mediated by the weak interaction. With the exception of the recent $D^{0} \bar{D}^{0}$-mixing measurements, which mostly constrain flavor mixing between first- and second-generation squarks [25], currently there are only upper bounds on charm or top FCNCs. Some of the most stringent limits on the flavor structure including the third generation come from $B$ - and $B_{s}$-meson measurements and involve the $b \rightarrow s$ and $b \rightarrow d$ quark transitions in meson mixing and decays. Particularly constraining are the radiative $B \rightarrow X_{s} \gamma$ and $B \rightarrow \rho \gamma$, semileptonic $B \rightarrow X_{s} \ell^{+} \ell^{-}$and $B \rightarrow \pi \ell^{+} \ell^{-}$decays and the $B_{d}-\bar{B}_{d}$ mass differences [26, 27, 28, 29, 30, 31, 32, 33, 34, 35].

Even with the strong current constraints from flavor physics taken into account, the MSSM beyond MFV has regions of parameter space where the couplings of a charged Higgs to light quarks are substantially modified by SUSY loops. For small $\tan \beta$ charged-Higgs searches at the LHC are a sensitive probe of supersymmetric flavor physics, in a similar way to rare decays at $B$ factories: they will never guarantee charged-Higgs discovery, but their experimental verification would shed light on otherwise poorly constrained aspects of the MSSM flavor sector, linked to the physics of supersymmetry breaking.

The paper is organized as follows: in Section \we study the single-charged-Higgs production $q \bar{q}^{\prime} \rightarrow H^{ \pm}$in the MSSM, assuming MFV and allowing for general flavor violation. A brief discussion of flavor violation in supersymmetric models is included in this section. We improve on earlier work [36] by a more general treatment of squark mixing and by taking into account FCNC constraints. In Section [II] we discuss current constraints on soft-breaking parameters from flavor-physics data and theory. In Section [V] we calculate charged-Higgs production rates in association with a hard jet, within and beyond MFV. A brief background study for the LHC environment is included. We summarize in Section $\mathrm{V}$ and provide details about flavored quarks and squarks in the appendix.

\section{SINGLE-CHARGED-HIGGS PRODUCTION}

We start by considering single-charged-Higgs production from quark-antiquark scattering at the LHC. To leading order this process can be described by a general type-II two-Higgs-doublet model. In Fig. 1 we show the Drell-Yan-like diagram for $q \bar{q}^{\prime} \rightarrow H^{ \pm}$. In the quark mass basis the corresponding coupling is given by

$$
\mathcal{L}_{H^{ \pm} q q^{\prime}}=\sqrt{2} V_{i j} \bar{u}_{i}\left(\frac{m_{d_{j}}}{v} P_{R} \tan \beta+\frac{m_{u_{i}}}{v} P_{L} \cot \beta\right) d_{j} H^{+}+\text {h.c. }
$$

with the quark fields $u, d$, their masses $m_{u, d}$ and the CKM matrix elements $V_{i j}(i, j=1, \ldots, 3)$. The Yukawas are given in terms of $v=2 m_{W} / g=246 \mathrm{GeV}, g=e / \sin \theta_{w}$. Here $\tan \beta=v_{u} / v_{d}=\left\langle H_{u}^{0}\right\rangle /\left\langle H_{d}^{0}\right\rangle$ denotes the ratio of vacuum expectation values of the two Higgs doublets. The physical charged Higgs scalar in terms of interaction eigenstates is $H^{+}=\sin \beta\left(H_{d}^{-}\right)^{*}+\cos \beta H_{u}^{+}$. The chiral projectors are defined as $P_{L, R}=\left(1 \mp \gamma_{5}\right) / 2$.

Following Eq.(11) the amplitude for single-Higgs production in the type-II two-Higgs-doublet model is proportional to the quark Yukawa, i.e. it is small unless third-generation quarks are involved. This chiral suppression is generic and with proper assumptions survives radiative corrections, like the SUSY-QCD corrections shown 

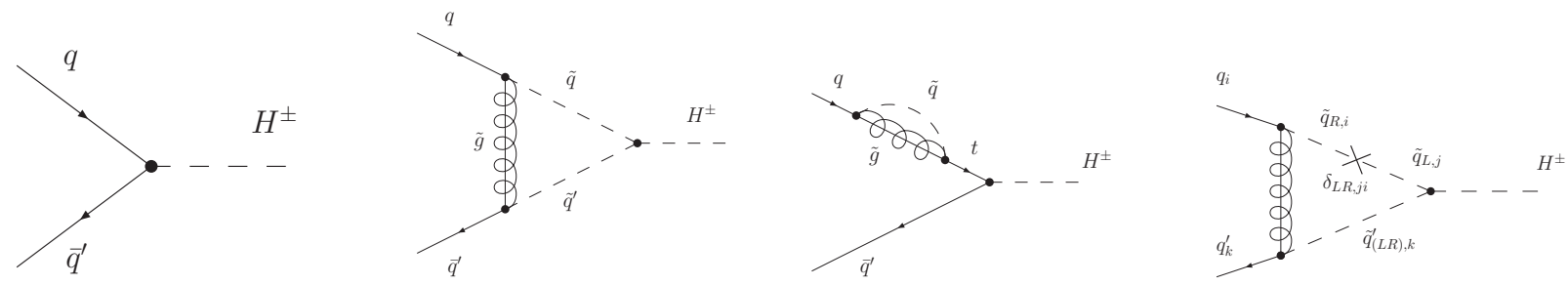

FIG. 1: Feynman diagrams contributing to $q \bar{q}^{\prime} \rightarrow H^{ \pm}$in the MSSM at tree level and at one-loop level. The last diagram is shown only to illustrate the contributions arising in SUSY models beyond MFV. Instead of the mass insertion approximation, we use the complete squark-mass matrix for the numerical analyses throughout the paper.

in Fig. 1. Every gauge-invariant operator linking quark-antiquark-Higgs fields involves a chirality flip, hence vanishes with $m_{q} \rightarrow 0$ as long as the theory has a chiral limit. The renormalizable operators contributing up to dimension 4 are (modulo hermitian conjugates) [37]

$$
\bar{Q} H_{u}^{C} U, \quad \bar{Q} H_{d}^{C} D, \quad \bar{Q} H_{d} U, \quad \bar{Q} H_{u} D,
$$

where $H^{C}=i \tau_{2} H^{*}$, and $Q$ and $U, D$ are the $S U(2)$ weak-interaction eigenstate doublets and singlets, respectively. In general, capital letters describe interaction eigenstates, while small letters denote fermionic mass eigenstates.

While the first two operators in Eq.(2) are the usual tree-level Yukawa interactions, the second two operators involve the 'wrong' Higgs fields, and do not occur in the plain type-II two-Higgs-doublet model. Such 'wrong' Higgs operators are induced by SUSY breaking. They are proportional to a soft SUSY-breaking parameter like the gluino mass or an $A$-term and couple the Higgs to a squark loop [18]. Since after spontaneous symmetry breaking all operators in Eq.(2) contribute to the fermion masses, the lowest-order relation between the quark masses and the Yukawas is broken. This effect becomes numerically important for large $\tan \beta$. Since we are only interested in small and moderate $\tan \beta$, we can safely neglect this effect. Wrong Higgs couplings increase also with increasing $\mu$ term [18, 19]. As far as the chiral limit of the MSSM is concerned, it is not spoiled as long as the soft-breaking $A^{u, d}$ terms are proportional to the respective quark Yukawa $Y^{u, d}$. Sometimes, this proportionality is made explicit by rescaling the $A^{u, d}$ terms and splitting off the Yukawa matrix as a prefactor.

For single-Higgs production we are limited to the four operators in Eq.(2), including necessarily some kind of chirality flip. We can build an extended set of operators to contain fermions of the same chirality by simply adding an external gauge field. We will entertain this possibility in Section IV.

\section{A. Tree-Level Single-Higgs Production}

Because the top quark is too heavy for the gluon to split into a collinear $t \bar{t}$ pair at the LHC, the large flavor-diagonal CKM element $V_{t b}$ does not play any role in single-charged-Higgs production. Instead, in a twoHiggs-doublet model all interactions in Eq.(11) suffer suppression either from light-flavor quark masses or from quark mixing, parameterized by CKM entries such as $V_{c b} \simeq 0.04$ [36]. Modulo differences in parton densities, from Eq.(11) we expect the largest production rates from bottom-charm fusion or strange-charm fusion, since $m_{s} V_{c s}$ and $m_{b} V_{c b}$ are of similar size. Using the $\overline{\mathrm{MS}}$ quark masses given in Table (A-13) at typical Higgs-mass scales we find explicitly that the charm-bottom channel is favored. Hence, for large enough values of $\tan \beta$ the biggest contribution to the single-charged-Higgs production cross section will always be proportional to $\left|m_{b} V_{c b} \tan \beta\right|^{2}$.

For example, for $\tan \beta=7$ and a charged-Higgs mass of $m_{H^{ \pm}}=188 \mathrm{GeV}$ we find LHC cross sections for $H^{+}$production of $\sigma_{c s}=10.1 \mathrm{fb}$ and $\sigma_{c b}=25.3 \mathrm{fb}$. If we neglect the theoretically poorly defined strange-quark Yukawa, the cross section decreases to $\sigma_{c s}=0.56 \mathrm{fb}$. Neglecting the charm Yukawa does not visibly shift $\sigma_{c b}$. The more we then increase $\tan \beta$, the more we will be dominated by the enhanced bottom Yukawa in $\bar{b}-c$ scattering, in spite of its strong CKM suppression.

The charged Higgs can best be found in $H \rightarrow \tau \nu$ decays. In general, charged-Higgs decays are very similar to $W$ decays, with a bias towards heavy fermions, because of the Yukawa instead of the generation-universal 
gauge couplings. The irreducible background to our searches is single- $W$ production, mediated by

$$
\mathcal{L}_{W^{ \pm} q q^{\prime}}=-V_{i j} \frac{g}{\sqrt{2}} \bar{u}_{i} \gamma^{\mu} P_{L} d_{j} W_{\mu}^{+}+\text {h.c.. }
$$

This coupling is much bigger than the couplings in Eq.(1): $g / \sqrt{2} \sim \mathcal{O}(0.5) \gg Y^{u, d}$. Hence, the $W^{+}$production cross section of $90 \cdot 10^{6} \mathrm{fb}$ will be a serious challenge to our $H^{+}$search in the two-Higgs-doublet model. Applying a phase-space cut on the transverse mass $m_{T}$ of the $\mathrm{W}$ boson between the Jacobian peaks from $W^{ \pm}$and $H^{ \pm}$ production, which appear at $m_{T}=m_{W}$ and $m_{T}=m_{H^{+}}$, respectively, reduces the $\mathrm{W}$ production cross section by a factor of $10^{2}$ to $10^{3}$. This drastic background reduction is still not enough for a significant signal/background ratio on the basis of integrated cross sections. In practice, one thus has to investigate whether a shoulder from the Higgs Jacobian peak can be resolved in the $\mathrm{W}$ transverse mass spectrum. The corresponding significance and further background suppression in this spectrum can only be seriously investigated by including detector effects such as efficiencies and momentum smearing, a task that we have to leave with experimental experts.

\section{B. Loop-Induced Single-Higgs Production in the Flavored MSSM}

Not assuming MFV has serious impact on the production rate for $q \bar{q}^{\prime} \rightarrow H^{ \pm}$. Squark loops will weaken the CKM suppression at the charged-Higgs-bottom vertex through flavor mixing. The squark mixing matrix collects $D$ and $F$ terms, and soft terms from the SUSY-breaking Lagrangian defined in Eq.(A-7), the latter being susceptible to flavor violation beyond MFV.

The hermitian $6 \times 6$ squark mass matrices $\mathcal{M}_{q}^{2}$ for up- and down-type squarks are composed out of the leftand right-handed blocks $M_{q A B}^{2}(A, B=L, R)$. Each block is a $3 \times 3$ matrix in generation space:

$$
\mathcal{M}_{q}^{2}=\left(\begin{array}{ll}
M_{q L L}^{2} & M_{q L R}^{2} \\
M_{q L R}^{2 \dagger} & M_{q R R}^{2}
\end{array}\right), \quad q=u, d .
$$

The explicit expressions for the $M_{q A B}^{2}$ are given in Eq. (A-9) and A-10. Following the quark notation, doublet squarks are labeled as $L$, as opposed to $S U(2)$ singlets, which are marked as $R$. Squark mass matrices are given in the basis defined by diagonal quark Yukawas (super-CKM basis).

$R$-parity-conserving effects beyond MFV are confined to the soft-breaking Lagrangian. Hence, if we assume MFV the off-diagonal elements of the sub-blocks vanish in the super-CKM basis-modulo tiny effects from renormalization-group running [38], that is, $M_{q L L i j}^{2} \propto m_{0}^{2} \delta_{i j}, M_{q R R i j}^{2} \propto m_{0 q}^{\prime 2} \delta_{i j}$ and $M_{q L R i j}^{2} \propto m_{q_{i}} A_{0} \delta_{i j}$. The SUSY-breaking mass parameters are the generation-universal SUSY-breaking scalar masses $m_{0}^{2}, m_{0 q}^{\prime 2}$ and the trilinear term $A_{0}$.

To trace back and discuss the sources of new-physics flavor violation, it is useful to define the dimensionless mass insertions [22, 23]

$$
\delta_{A B, i j}^{q} \equiv \frac{M_{q A B i j}^{2}}{\tilde{m}^{2}} .
$$

The denominator is the geometric mean $\tilde{m}^{2}=m_{A i i} m_{B j j}$ of the squared scalar masses of $\tilde{q}_{A i}$ and $\tilde{q}_{B j}$. Following the previous discussion, the off-diagonal entries of $\delta_{A B i j}^{q}, i \neq j$ are significant only in non-MFV models and can be complex, inducing $\mathrm{CP}$ violation. We confine ourselves to real $\delta_{A B}^{q}$. Note that in our numerical calculations we diagonalize the squark mass matrices and do not employ a perturbative expansion in the $\delta_{A B}^{q}$, which would avoid the calculation of the squark unitary transformations [22]. We only use the intuitive mass-insertion approximation for illustration and order-of-magnitude estimates, see also the appendix of Ref. [39] for formulae.

For our analysis of charged-Higgs production involving squark loops the three-scalar couplings of squarks and Higgses are relevant. They stem from three different sources:

$$
\mathcal{L}_{H^{ \pm} \tilde{q} \tilde{q}^{\prime}}=D \text {-term }+F \text {-term }+A \text {-term. }
$$

The $D$ term couples the charged Higgs to two doublet squarks, i.e. the combination $L L$ :

$$
\left.\mathcal{L}_{H^{ \pm}} \tilde{q} \tilde{q}^{\prime}\right|_{D}=-\frac{V_{i j} g m_{W}}{\sqrt{2}} \sin (2 \beta) \tilde{u}_{L i}^{*} \tilde{d}_{L j} H^{+}+\text {h.c.. }
$$


This $D$-term contribution is proportional to $\sin (2 \beta)$, i.e. it is suppressed by $1 / \tan \beta$ for large tan $\beta$. Most importantly, it does not break chirality.

While $D$ terms are gauge couplings, $F$ terms arise from the superpotential. $F$-term couplings of squarks to Higgses are Yukawa induced and involve all four possible combinations of $L$ and $R$ squarks:

$$
\begin{aligned}
\left.\mathcal{L}_{H^{ \pm} \tilde{q} \tilde{q}^{\prime}}\right|_{F}=\frac{g V_{i j}}{\sqrt{2} m_{W}} H^{+} & {\left[\tilde{u}_{L, i}^{*} \tilde{d}_{L, j}\left(m_{d, j}^{2} \tan \beta+m_{u, i}^{2} \cot \beta\right)\right.} \\
& \left.+\tilde{u}_{R, i}^{*} \tilde{d}_{R, j} m_{u, i} m_{d, j}(\cot \beta+\tan \beta)+\mu m_{d, j} \tilde{u}_{L, i}^{*} \tilde{d}_{R, j}+\mu m_{u, i} \tilde{u}_{R, i}^{*} \tilde{d}_{L, j}\right] .
\end{aligned}
$$

$A$-terms and soft masses are general soft SUSY-breaking parameters. $A$-terms occur with a chirality-flipping squark combination. We keep the soft terms $A^{u, d}$ with all flavor indices $i, j, k$ and without a Yukawa prefactor:

$$
\left.\mathcal{L}_{H^{ \pm} \tilde{q} \tilde{q}^{\prime}}\right|_{A}=\tilde{d}_{L i} V_{k i} A_{k j}^{u} \tilde{u}_{R j}^{*} \cos \beta H^{+}+\tilde{u}_{L i} V_{i k}^{*} A_{k j}^{d} \tilde{d}_{R j}^{*} \sin \beta H^{-}+\text {h.c.. }
$$

Both $D$ - and $F$-term contributions to the charged-Higgs-squark coupling are driven by the respective CKM element, as a result of being MFV. This is different for the A-terms induced by SUSY breaking. We note that our MSSM Lagrangian is defined at the weak scale, so all parameters are evaluated at the scale of the charged-Higgs mass.

We address the question of how large the $H^{ \pm}$production cross sections in the MSSM can be with general flavor after taking into account experimental and theoretical constraints. The dominant one-loop corrections are due to the gluino vertex and self-energy diagrams shown in Fig. 1 at $\mathcal{O}\left(\alpha_{s}\right)$, having the largest gauge couplings. Beyond MFV, the loop diagrams do not have to include a quark mass to yield a chiral operator. Instead, we can, for example, combine a gaugino mass with a left-right mixing $\delta_{L R}$ among the squarks. This combination can lift the supersymmetric charged-Higgs production rate above the two-Higgs-doublet model prediction, despite its loop suppression.

We are mainly interested in mixing in the up-squark sector, because here bigger beyond-Standard-Model effects are possible. As it turns out, the leading contribution to charged-Higgs production involves $\tilde{t}_{L}-\tilde{u}(c)_{R}$ mixing rather than $\tilde{t}_{R}-\tilde{u}(c)_{L}$ : while the latter can have particularly big impact on rare $K$ and $B$ decays through a modified FCNC $Z$-boson vertex [30, 39], the former escapes these constraints, as we will explain in Section II]. Contributions not involving a third-generation squark are negligible.

We first give order-of-magnitude estimates for $H^{ \pm}$production from the gluino loop versus the tree-level strange-charm $\mathcal{A}_{c s}$ and bottom-charm $\mathcal{A}_{c b}$ amplitude discussed in the previous section:

$$
\begin{array}{ll}
\frac{\mathcal{A}_{\text {gluino-loop }}}{\mathcal{A}_{c s}} \propto \frac{\alpha_{s}}{4 \pi} \frac{m_{\tilde{g}}}{m_{c}} \delta_{L R, 3 i}^{u}, & \\
\frac{\mathcal{A}_{\text {gluino-loop }}}{\mathcal{A}_{c b}} \propto \frac{\alpha_{s}}{4 \pi} \frac{m_{\tilde{g}}}{V_{c b} m_{b}} \frac{1}{\tan ^{2} \beta} \delta_{L R, 3 i}^{u}, & i=1,2 .
\end{array}
$$

For these ratios we approximate the diagonal CKM elements $V_{t b}, V_{c s} \simeq 1$. Both ratios in Eq. (10) exhibit an enhancement of the gluino loop that can be as large as $\mathcal{O}(10)$ for suitable SUSY masses and tan $\beta$. Depending on the initial state, up $(i=1)$ or charm $(i=2)$ quarks can induce such a genuine MSSM contribution.

With this estimate in mind we then calculate $H^{+}$production from quark-antiquark fusion including the dominant squark-gluino loops. Generally, the amplitude $\mathcal{A}^{i j}$ for $u_{i} \bar{d}_{j} \rightarrow H^{+}$production can be written with quark $u_{q}$ and antiquark $v_{q}$ spinors as

$$
\mathcal{A}^{i j}=\sum_{\sigma} \mathcal{F}^{i j, \sigma} \mathcal{M}^{i j, \sigma} \quad \text { with } \quad \mathcal{M}^{i j, \sigma}=\bar{v}_{d_{j}} P_{\sigma} u_{u_{i}}, \quad \mathcal{F}^{i j, \sigma}=\mathcal{F}_{0}^{i j, \sigma}+\mathcal{F}_{S}^{i j, \sigma}+\mathcal{F}_{V}^{i j, \sigma}, \quad \sigma=L, R .
$$

We obtain for the tree-level contribution $\mathcal{F}_{0}$ and to leading order in the mass insertion expansion for the one-loop self-energy $\mathcal{F}_{S}$ and vertex $\mathcal{F}_{V}$ contributions

$$
\begin{aligned}
\mathcal{F}_{0}^{i j, R} & =\frac{e V_{i j}^{*}}{\sqrt{2} m_{W} \sin \theta_{w}} m_{u_{i}} \cot \beta, \\
\mathcal{F}_{0}^{i j, L} & =\frac{e V_{i j}^{*}}{\sqrt{2} m_{W} \sin \theta_{w}} m_{d_{j}} \tan \beta, \\
\mathcal{F}_{S}^{i j, R} & =\frac{\sqrt{2} e V_{3 j}^{*}}{m_{W} \sin \theta_{w}} \frac{\alpha_{s}}{4 \pi} C_{F} \frac{m_{\tilde{g}}}{\tan \beta} \delta_{L R, 3 i}^{u} \tilde{m}^{2} \mathcal{I}_{12}\left(m_{\tilde{g}}, m_{\tilde{q}}\right), \\
\mathcal{F}_{V}^{i j, R} & =\frac{\sqrt{2} e V_{3 j}^{*}}{m_{W} \sin \theta_{w}} \frac{\alpha_{s}}{4 \pi} C_{F}\left(\frac{m_{t}^{2}}{\tan \beta}-m_{W}^{2} \sin (2 \beta)\right) m_{\tilde{g}} \delta_{L R, 3 i}^{u} \tilde{m}^{2} \mathcal{I}_{13}\left(m_{\tilde{g}}, m_{\tilde{q}}\right),
\end{aligned}
$$



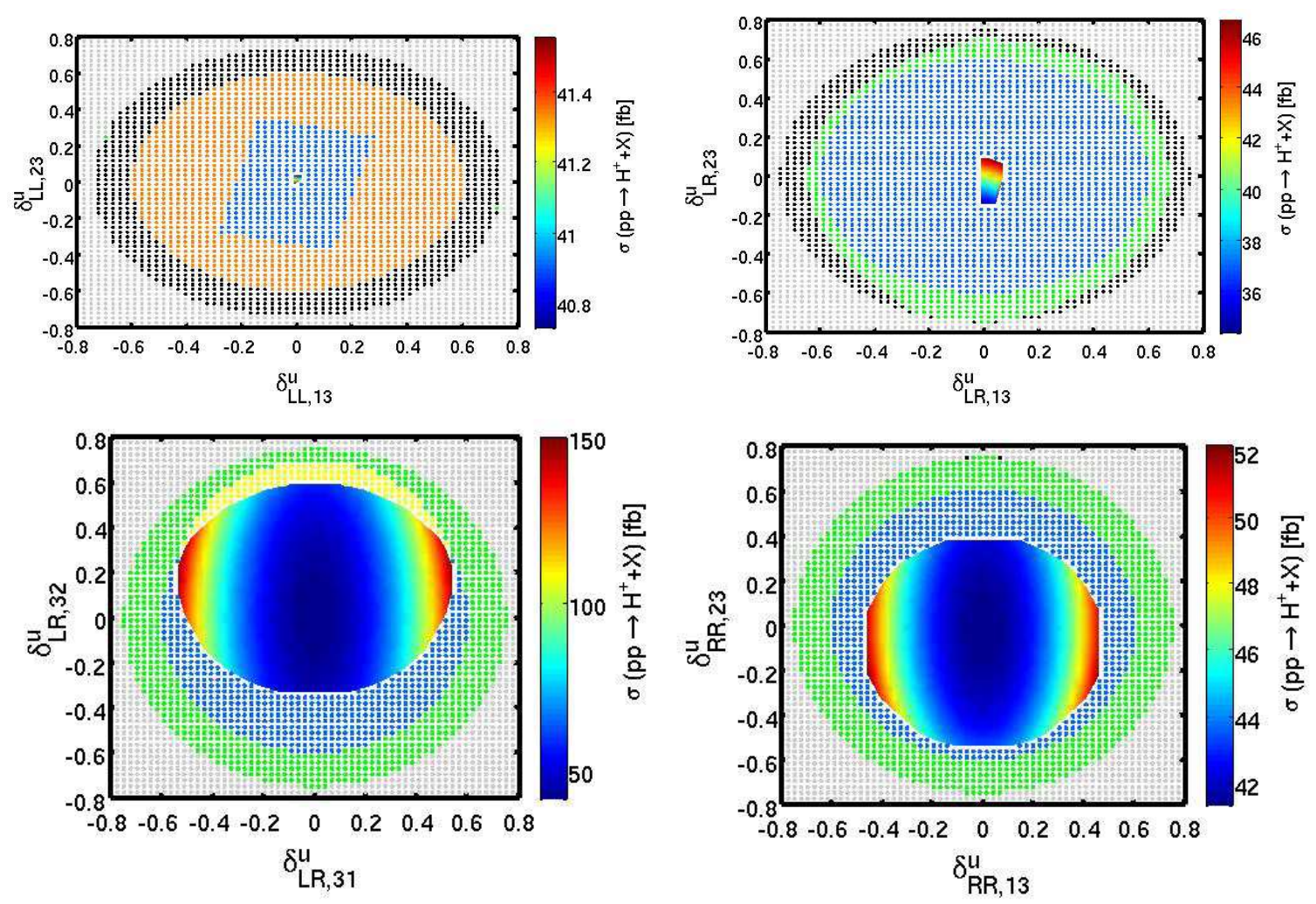

FIG. 2: Single-charged-Higgs production cross sections at the LHC. In the rainbow-colored area we include beyond-MFV parameters around the lower-mass parameter point (14). Two $\delta_{A B, i j}^{u}$ are varied in each panel, all others are set to zero. The area outside the rainbow is ruled out experimentally.

where we define

$$
\mathcal{I}_{l m}\left(m_{\tilde{g}}, m_{\tilde{q}}\right)=\int \frac{d^{4} q}{i \pi^{2}} \frac{1}{\left(q^{2}-m_{\tilde{g}}^{2}\right)^{l}\left(q^{2}-m_{\tilde{q}}^{2}\right)^{m}}, \quad l+m>2
$$

Here, $m_{\tilde{q}}$ denotes a generic squark mass scale in the loops. Note that the functions $\mathcal{I}_{l m}$ scale as $M_{S U S Y}^{4-2 l-2 m}$ for $M_{S U S Y} \sim m_{\tilde{g}} \sim m_{\tilde{q}}$. The left-chiral contributions $\mathcal{F}_{S, V}^{i j, L}$ vanish if all quarks but the top quark are massless. For bottom-up fusion Eqs. (12) show explicitly that the gluino loops with $\delta_{L R, 3 i}^{u}$ are proportional to $V_{t b} m_{\tilde{g}}$, hence avoid the CKM and quark-mass suppression present in the non-SUSY amplitudes. We note the cancellation of $F$-term $\left(\propto m_{t}^{2}\right)$ and $D$-term $\left(\propto m_{W}^{2}\right)$ contributions in the vertex correction $\mathcal{F}_{V}^{i j, R}$. Therefore, the self-energies give the dominant MSSM contribution with parametric dependence as in Eq.(10). Our analytical formulae are in agreement with Ref. [36], where only stop-scharm mixing in $A$-terms has been considered.

As already stressed, we do not use the mass-insertion series in our numerical analysis presented in the next section, but diagonalize the full squark mass matrix. We also investigate effects of $L L$ and $R R$ squark mixing with stops. Specifically we use the program FeynArts [40] for the generation of graphs and amplitudes, the package FormCalc/LoopTools [41] for their evaluation, and the program HadCalc [42] for the convolution with the CTEQ6 43] parton distribution functions. Parts of the calculations have been checked with in-house routines.

\section{Supersymmetric Parameter Space beyond MFV}

To test the effects of flavor structures on the single-Higgs cross section we start with a generic MFV SUSY parameter point which does not violate any current bounds. We then allow for flavor violation beyond MFV, as illustrated by $\delta_{A B}^{q}$ as defined in Eq.(5). Because of current experimental and theoretical constraints discussed 
in detail in Section III the up-squark parameters $\delta_{L R, 3 i}^{u}$ and $\delta_{R R, 3 i}^{u}$ involving 1-3 and 2-3 mixing are the least constrained and therefore expected to cause the biggest effects. An insertion of $\delta_{L R, j i}^{q}$ is illustrated in the last Feynman diagram of Fig. 1. Specifically, we are dealing here with gluino-squark loop contributions to $u \bar{b} \rightarrow H^{+}$ and $c \bar{b} \rightarrow H^{+}$processes, which are not CKM suppressed by means of the genuine SUSY flavor breaking parameters $\delta_{3 i}^{u}$. Incoming first- and second-generation quarks have larger luminosities, but supersymmetric loop effects are suppressed by small squark-mixing couplings such as $\delta_{L R 11}^{u, d}$ and $\delta_{L R 22}^{u, d}$.

Our starting (lower-mass) parameter point is given by:

$$
\begin{aligned}
& \tan \beta=7, \quad m_{A}=170 \mathrm{GeV}, \quad \mu=-300 \mathrm{GeV}, \\
& m_{\tilde{U}_{L L, R R} i i}=m_{\tilde{D}_{L L, R R} i i}=600 \mathrm{GeV}, \quad M_{2}=700 \mathrm{GeV}, \quad m_{\tilde{g}}=500 \mathrm{GeV} \text {, } \\
& A^{u, c}=0, \quad A^{d, s, b}=0, \quad A^{t}=1460 \mathrm{GeV},
\end{aligned}
$$

where $m_{A}$ denotes the mass of the CP-odd Higgs leading to $m_{H^{+}}=188 \mathrm{GeV} . M_{2}$ is the SUSY-breaking wino mass. The diagonal soft-breaking entries in the squark mass matrices defined in Eq. (A-7) is chosen universal. All parameters are given at a scale of order $m_{H^{+}}$. The large value of $A^{t}$ (corresponding to $\delta_{L R, 33}^{u}$ ) increases the light Higgs mass to $119.9 \mathrm{GeV}$ at two loops [46]. For this parameter choice the tree-level $\mathrm{H}^{+}$production cross section at the LHC in the two-Higgs-doublet model is $41.2 \mathrm{fb}$.

The production cross sections as a function of the dominant beyond-MFV mass insertions in the up-sector are shown in Fig. 2. Beyond-MFV effects can enhance the single-Higgs rate to values above $100 \mathrm{fb}$. The size of the production cross section is encoded in the rainbow scale in all panels of Fig. 2, while the parameter choices outside this area are ruled out. We will discuss the constraints in more detail in Section III. The different experimental constraints impacting the (lower-mass) parameter point shown in Fig. 2 include:

- Tevatron searches for mass-degenerate first- and second-generation squarks put constraints on their masses [44]. The D $\varnothing$ analysis has been performed within minimal supergravity, but assuming similar decay chains the mass bounds hold in a general MSSM context. In our analysis we require $m_{\tilde{q}}>200 \mathrm{GeV}$. This rules out the yellow points.

- Squark searches and radiative and semileptonic $B$-decay limits rule out the green points.

- Black points are forbidden by the squark-mass limits, $B$ mixing, and radiative and semileptonic $B$ decays.

- Blue points indicate a violation of the radiative and semileptonic $B$ decay bounds only.

- Orange points correspond to a violation of the $B$ mixing and radiative and semileptonic decay limits.

- Red points are ruled out by $B$ mixing alone.

- Grey points on the outside of the panels indicate a negative squark mass square after diagonalizing the squark mass matrix.

In Fig. 2 we see that the limits on radiative and semileptonic decays followed by the Tevatron limit on lightflavor squark masses define two distinct boundaries of forbidden parameter space. After taking into account all limits, the off-diagonal entry $\delta_{L R, 31}^{u}$ has the strongest impact on the rate. It yields a maximal single-Higgs rate for $\left|\delta_{L R, 31}^{u}\right| \sim 0.6$ (third panel). The effect of $\delta_{L R, 32}^{u}$ is similar to $\delta_{L R, 31}^{u}$, except that the process now requires an incoming $c_{R}$. The latter is disfavored with respect to incoming $u_{R}$ by smaller parton luminosity. Another MFV pattern that leads to an enhanced production rate is $\left|\delta_{R R, 13}^{u}\right| \sim 0.5$ (fourth panel). This contribution requires a further $L R$ switch through the squarks, which could be an $A$ - or $F$-term squark- $H^{ \pm}$coupling. Since $A_{33}^{u}$ is typically large (see Eq.(19)), the relevant combination $\delta_{R R, 13}^{u} \delta_{L R, 33}^{u}$ is numerically sizeable, as is the $F$-term contribution $\propto m_{t} \mu \delta_{R R, 13}^{u}$.

We recall that for the numerical analysis we do not use mass insertions. Otherwise, values of $\delta_{A B, i j}^{u}$ close to unity would not give numerically reliable predictions. Current experimental limits, for example from squark searches generally imply $\delta^{u}<1$, but not necessarily $\delta^{u} \ll 1$.

In Fig. 3 we show the ratio of the cross section including beyond-MFV diagrams over the (tree-level) twoHiggs-doublet-model cross section. At tree level we include all Standard-Model Yukawas. For the different curves we vary the charged-Higgs mass between 188 and $500 \mathrm{GeV}$ and find little impact on the relative size of 


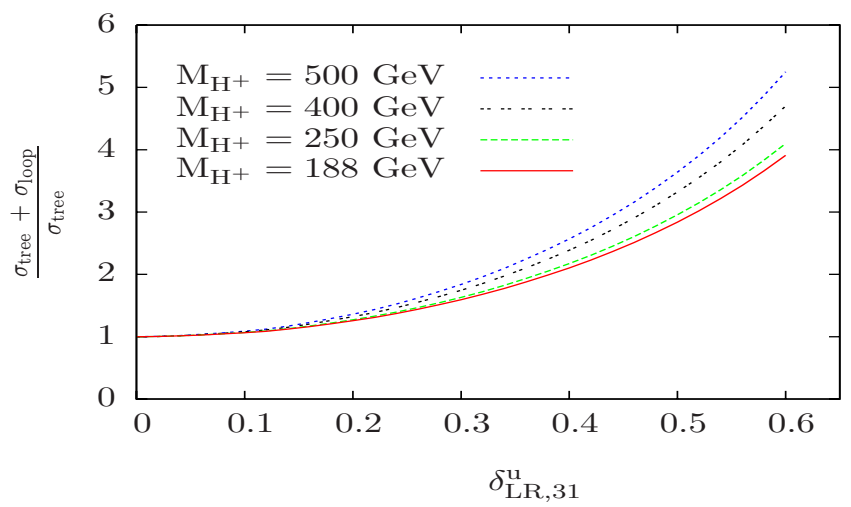

FIG. 3: Ratio of single-charged-Higgs cross sections including supersymmetric beyond-MFV loops vs. in the two-Higgsdoublet model. All supersymmetric parameters are given in Eq.(14). All beyond-MFV parameters except for $\delta_{L R, 31}^{u}$ are zero.

the contributions. All supersymmetric parameters correspond to the lower-mass parameter choice (14). To show the typical size of the observed effect, we vary the dominant beyond-MFV parameter $\delta_{L R, 31}^{u}$ within its allowed range, with all other beyond-MFV parameters zero. While beyond-MFV diagrams are formally of higher order, namely supersymmetric one-loop corrections, we can already read off Eq. (10) that they lead to larger effects. This is indeed confirmed by Fig. 3. Supersymmetric corrections by factors of $\mathcal{O}(5)$ are not a reason to worry about the stability of perturbation theory. Instead, they reflect an additional source of fermionic mass insertions, which can be large compared to five light-flavor Yukawas, as discussed at the beginning of this section.

The impact of the experimental squark bound depends crucially on the squark masses we choose. For an illustration we consider the eigenvalues $m_{i}^{2}$ of a $(2 \times 2)$ mass matrix with off-diagonal mixing $\delta$ and a diagonal sfermion mass $m_{0}$ :

$$
M^{2}=m_{0}^{2}\left(\begin{array}{cc}
1 & \delta \\
\delta & \Delta
\end{array}\right), \quad m_{i}^{2}=m_{0}^{2}\left(\frac{1+\Delta}{2} \pm \sqrt{\frac{(1-\Delta)^{2}}{4}+\delta^{2}}\right) .
$$

We also allow for non-degenerate diagonal entries $\Delta$ not too far from one (as possible in models beyond MFV). Both $\delta$ and $1-\Delta$ increase the mass splitting. From an experimental limit $m_{i}>m_{\text {bound }}$ we obtain a bound on $\delta$ as a function of $m_{0}$ :

$$
\delta<\sqrt{\left(1-r^{2}\right)\left(\Delta-r^{2}\right)}, \quad r=\frac{m_{\text {bound }}}{m_{0}}<1, \Delta
$$

or simply $\delta<1-r^{2}$ for degenerate diagonal matrix elements. For $\Delta<1(\Delta>1)$, the constraint on the mixing $\delta$ improves (eases) with respect to the $\Delta=1$ case. Clearly, for increasing values of the squark mass scale $m_{0}$ the bound on the off-diagonal mixing from direct search limits weakens and the flavor constraints having a different decoupling behaviour are of most importance.

We can make this explicit by slightly increasing the soft-breaking squark masses and $m_{A}$, which gives us another (higher-mass) parameter point:

$$
\begin{aligned}
& \tan \beta=5, \quad m_{A}=500 \mathrm{GeV}, \quad \mu=-200 \mathrm{GeV}, \\
& m_{\tilde{U}_{L L, R R} i i}=m_{\tilde{D}_{L L, R R} i i}=800 \mathrm{GeV}, \quad M_{2}=500 \mathrm{GeV}, \quad m_{\tilde{g}}=500 \mathrm{GeV}, \\
& A^{u, c}=0, \quad A^{d, s, b}=0, \quad A^{t}=1260 \mathrm{GeV} .
\end{aligned}
$$

The charged-Higgs mass is now $m_{H^{+}}=507 \mathrm{GeV}$. The tree-level cross section of $0.48 \mathrm{fb}$ in the two-Higgs-doublet model is suppressed by this heavy final-state mass. The color coding for the different constraints in Fig. 4 is the same as in Fig. 2, The basic features of the higher-mass parameter point and the previously discussed lower-mass parameter point are similar. The effects of the squark-mixing parameters $\delta_{L R, 3 i}^{u}$ and $\delta_{R R, i 3}^{u}$ can be

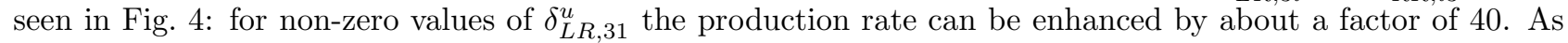
before, rare $B$ decays strongly limit the parameter space, complemented by similarly strong limits from the 

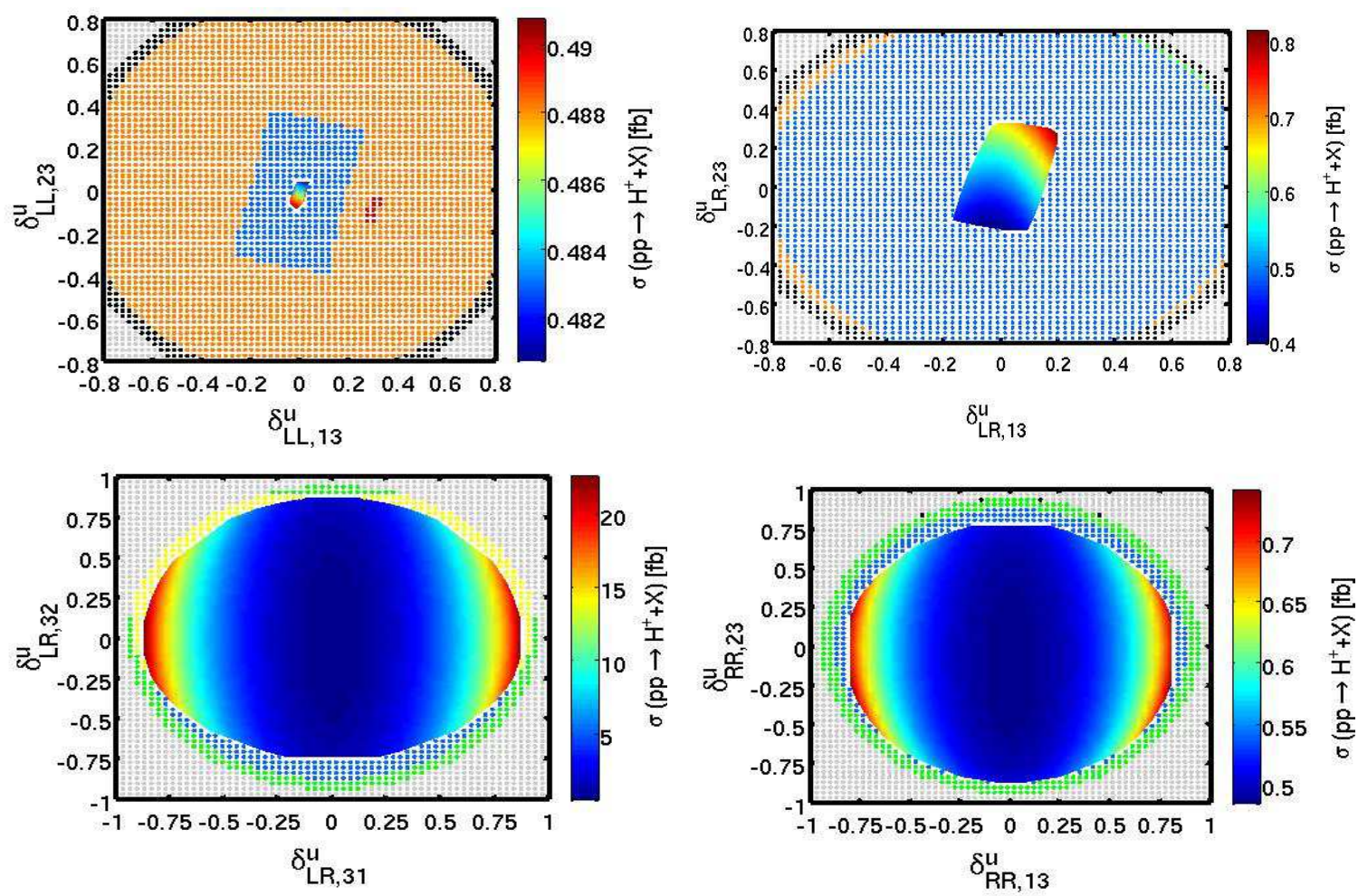

FIG. 4: Single-charged-Higgs production cross sections at the LHC. In the rainbow-coded area we include beyond-MFV parameters around the higher-mass parameter point (17). Two $\delta_{A B, i j}^{u}$ are varied in each panel, all others are set to zero. The area outside is ruled out.

direct searches at the Tevatron. The main difference compared to the low-mass point is the size of the allowed region. Instead of a typical value of $\delta^{u} \lesssim 0.5$ for $600 \mathrm{GeV}$ squark masses with heavier squarks we can have bigger mixing $\delta^{u} \lesssim 0.8$. Note that the shift in the charged-Higgs production including flavor structures beyond MFV from the parameters of Eq.(14) to Eq.(17) is mostly due to the heavier Higgs mass.

\section{FLAVOR PHYSICS CONSTRAINTS}

The vast number of past and ongoing flavor-physics measurements has serious impact on flavor physics at the LHC. From the previous section and the rough estimate in Eq.(10) it is obvious that without any constraints on squark mixing the charged-Higgs production rates could be arbitrarily large. However, flavor physics strongly constrains the structure of the general squark matrices in Eq.(44). The important parameters are the $L R$ entries induced by the $A$-terms $A^{u, d}$ and the corresponding $(L L, R R)$-type mass matrices $m_{\tilde{U}_{L}, \tilde{D}_{L}, \tilde{U}_{R}, \tilde{D}_{R}}^{2}$, which occur at tree level in the SUSY-breaking Lagrangian in the super-CKM basis, which we write out in Eq. (A-7).

We summarize the theoretical and experimental constraints acting on the relevant flavored SUSY parameters:

- $A_{i i}^{u, d}$ : diagonal $A$-term entries contribute to up- and down-quark masses at one loop:

$$
\delta m_{q_{i}} \propto \frac{\alpha_{s}}{4 \pi} m_{\tilde{g}} \delta_{L R i i}^{q}, \quad(q=u, d ; \quad i=1,2,3) .
$$

For the exact dimensionless loop functions see e.g. Ref. [45]. We require perturbativity of SUSY-QCD corrections $\delta m_{q} \lesssim m_{q}$. This effectively limits the set of $A_{i i}^{u, d}=\delta_{L R i i}^{u, d} M_{S U S Y}^{2} / v_{u, d}$ to large values of $A_{33}$ only.

$-A_{33}^{u, d}$ : loop corrections lift the lighter MSSM Higgs mass from $m_{Z}$ to above the LEP2 limits. For fixed stop (and for very large $\tan \beta$ also sbottom) masses this translates into an upper bound [46]

$$
\left|A_{33}^{u}\right| \lesssim \mathcal{O}(3) y_{t} M_{\mathrm{SUSY}} .
$$


- $A_{13}^{u, d}, A_{23}^{u, d}, A_{31}^{u, d}, A_{32}^{u, d}$ : general vacuum stability constraints limit the inter-generational $A$-terms $[24]$ :

$$
\begin{aligned}
&\left|A_{i 3}^{d}\right|,\left|A_{3 i}^{d}\right| \leq \frac{m_{b}}{v_{d}} \sqrt{2 \tilde{m}(d)^{2}+\tilde{m}(\ell)^{2}} \simeq \sqrt{3} y_{b} M_{\mathrm{SUSY}}, \\
&\left|A_{i 3}^{u}\right|,\left|A_{3 i}^{u}\right| \leq \frac{m_{t}}{v_{u}} \sqrt{2 \tilde{m}(u)^{2}+2 \tilde{m}(\ell)^{2}} \simeq \sqrt{3} y_{t} M_{\mathrm{SUSY}}, \quad i=1,2 .
\end{aligned}
$$

The masses $\tilde{m}(u), \tilde{m}(d), \tilde{m}(\ell)$ are the mean squark and slepton masses defined for Eq.(55). Because of the smaller Yukawas the down sector is subject to much stronger constraints than the up sector. We do not explicitly show analogous bounds for $L R$ mixing among the first and second generations, which are strongly suppressed by the strange and charm Yukawas.

$-A_{23}^{u, d},\left(m_{\tilde{U}_{L}, \tilde{D}_{L, R}}^{2}\right)_{23}$ : mixing between the second and third generation in the up and in the down sector is constrained by $(b \rightarrow s)$-type measurements, like $B \rightarrow X_{s} \gamma[26,27,32]$ and $B \rightarrow X_{s} \ell^{+} \ell^{-}$[27, 29, 30, 32] at the $B$ factories and the $\bar{B}_{s}-B_{s}$ mixing mass difference $\Delta m_{s}$ from the Tevatron [33, 34, 35]. Using CDF data only the latter implies the $90 \%$ C.L. range

$$
0.56<\frac{\Delta m_{s}}{\Delta m_{s}^{\mathrm{SM}}}<1.44
$$

dominated by theory uncertainty. To include the constraints from $B \rightarrow X_{s} \gamma$ decays we demand 2.94 . $10^{-4}<\operatorname{BR}\left(B \rightarrow X_{s} \gamma\right)<4.14 \cdot 10^{-4}[26,27]$. For $\operatorname{BR}\left(B \rightarrow X_{s} \ell^{+} \ell^{-}\right)$we use the data averaged over electrons and muons for dilepton masses above $0.2 \mathrm{GeV}$, leaving us with $2.8 \cdot 10^{-6}<\mathrm{BR}\left(B \rightarrow X_{s} \ell^{+} \ell^{-}\right)<$ $6.2 \cdot 10^{-6}[32]$.

$-A_{13}^{u, d},\left(m_{\tilde{U}_{L}, \tilde{D}_{L, R}}^{2}\right)_{13}$ : similarly, mixing between the first and third generation in the up and the down sector is constrained by $b \rightarrow d$ transitions: $B \rightarrow \rho \gamma$ [28], $B \rightarrow \pi \ell^{+} \ell^{-}$decays [31] and $\Delta m_{d}$ in $\bar{B}_{d}-B_{d}$ mixing at $90 \%$ C.L. [32, 34, 35]:

$$
0.46<\frac{\Delta m_{d}}{\Delta m_{d}^{\mathrm{SM}}}<1.54
$$

The first signal of $b \rightarrow d \gamma$ transitions has recently been seen by BaBar and Belle in $B \rightarrow(\rho, \omega) \gamma$ decays [28]. At $90 \%$ C.L. we use $0.63 \cdot 10^{-6}<\mathrm{BR}\left(B^{0} \rightarrow \rho^{0} \gamma\right)<1.24 \cdot 10^{-6}$. For semileptonic decays there exists only an upper bound from $\operatorname{BaBar} \operatorname{BR}\left(B \rightarrow \pi \ell^{+} \ell^{-}\right)<9.1 \cdot 10^{-8}$ at $90 \%$ C.L. [31].

$-m_{\tilde{U}_{L}}^{2}$ and $m_{\tilde{D}_{L}}^{2}$ : because SUSY breaking respects the $S U(2)$ gauge symmetry, the doublet soft-breaking masses are identical. Using the definitions (A-8) in the super-CKM basis this means

$$
m_{\tilde{U}_{L}}^{2}=V \cdot m_{\tilde{D}_{L}}^{2} \cdot V^{\dagger} .
$$

Hence, universal $m_{\tilde{D}_{L} i j}^{2}=m_{0}^{2} \delta_{i j}$ implies $m_{\tilde{U}_{L} i j}^{2}=m_{0}^{2} \delta_{i j}$, and vice versa.

- Inter-generational mixing involving the third generation also affects the lightest Higgs mass and the $\rho$ parameter [48, 49]. However, the constraints from rare decays and direct squark searches are generally stronger [49].

Let us summarize the generic features of the above constraints: the bounds on down-squark matrices $A^{d}$ and $m_{\tilde{D}_{L, R}}^{2}$ are in general stronger than those for up-squark matrices $A^{u}$ and $m_{\tilde{U}_{L, R}}^{2}$. This is due to theoretical arguments such as Eq.(20) and existing data on kaon and $B$ FCNCs, which involve down-squark mixing via strongly coupling gluino loops. Particularly strong bounds follow from radiative FCNC decays on the chiralityflipping coupling $A^{d}$ due to an $m_{\tilde{g}} / m_{b}$ enhancement. Hence, we can limit our analysis to up-squark mixing between different generations while neglecting down-squark mixing, as long as it is not required by Eq.(23). Furthermore, mixing between first- and second-generation squarks is tightly constrained by $K$-physics, e.g. , [23, 39] and by the recent measurements of $D^{0} \bar{D}^{0}$ mixing [25]. We therefore investigate effects on charged-Higgs production from mixing involving the third-generation up-type squarks, parameterized by $\delta_{i 3}^{u},(i=1,2)$. Since we do not consider in this work CP violation in the MSSM Lagrangian electric dipole moments do not pose constraints on the (real) soft terms.

Among the up-squark parameters, $A_{i 3}^{u}$ and $m_{\tilde{U}_{L} i 3}^{2}$ are constrained by data on $b \rightarrow s$ and $b \rightarrow d$ transitions, as well as by the weak isospin relation (23). Note that we strictly use the convention $A_{i j}=A_{L_{i} R_{j}} \neq A_{j i}$. On the 


\begin{tabular}{|cc|ll|lll|lll|}
\hline$m_{H^{+}}$ & $\tan \beta$ & $\sigma_{2 \mathrm{HDM}}$ & $\sigma_{2 \mathrm{HDM}}^{\left(m_{s}=0\right)}$ & $\sigma_{\mathrm{MFV}}$ & $\sigma_{\mathrm{MFV}}^{\left(m_{s}=0\right)}$ & $\sigma_{\mathrm{MFV}}^{\left(m_{q}=0\right)}$ & $\sigma_{\mathrm{SUSY}}$ & $\sigma_{\mathrm{SUSY}}^{\left(m_{s}=0\right)}$ & $\sigma_{\mathrm{SUSY}}^{\left(m_{q}=0\right)}$ \\
\hline $188 \mathrm{GeV}$ & 3 & $2.5 \cdot 10^{-1}$ & $1.9 \cdot 10^{-1}$ & $2.6 \cdot 10^{-1}$ & $2.0 \cdot 10^{-1}$ & $6.7 \cdot 10^{-4}$ & $14.3 \cdot 10^{0}$ & $14.2 \cdot 10^{0}$ & $13.9 \cdot 10^{0}$ \\
$188 \mathrm{GeV}$ & 7 & $9.9 \cdot 10^{-1}$ & $6.0 \cdot 10^{-1}$ & $1.1 \cdot 10^{0}$ & $6.5 \cdot 10^{-1}$ & $1.5 \cdot 10^{-4}$ & $4.6 \cdot 10^{0}$ & $4.4 \cdot 10^{0}$ & $3.0 \cdot 10^{0}$ \\
$400 \mathrm{GeV}$ & 3 & $4.0 \cdot 10^{-2}$ & $3.0 \cdot 10^{-2}$ & $4.2 \cdot 10^{-2}$ & $3.2 \cdot 10^{-2}$ & $4.2 \cdot 10^{-4}$ & $2.4 \cdot 10^{0}$ & $2.4 \cdot 10^{0}$ & $2.3 \cdot 10^{0}$ \\
$400 \mathrm{GeV}$ & 7 & $1.6 \cdot 10^{-1}$ & $1.0 \cdot 10^{-1}$ & $1.7 \cdot 10^{-1}$ & $1.1 \cdot 10^{-1}$ & $9.1 \cdot 10^{-5}$ & $7.9 \cdot 10^{-1}$ & $7.3 \cdot 10^{-1}$ & $5.4 \cdot 10^{-1}$ \\
$500 \mathrm{GeV}$ & 3 & $2.0 \cdot 10^{-2}$ & $1.44 \cdot 10^{-2}$ & $2.1 \cdot 10^{-2}$ & $1.5 \cdot 10^{-2}$ & $3.5 \cdot 10^{-4}$ & $1.3 \cdot 10^{0}$ & $1.3 \cdot 10^{0}$ & $1.2 \cdot 10^{0}$ \\
$500 \mathrm{GeV}$ & 5 & $4.2 \cdot 10^{-2}$ & $2.7 \cdot 10^{-2}$ & $4.4 \cdot 10^{-2}$ & $2.9 \cdot 10^{-2}$ & $1.4 \cdot 10^{-4}$ & $5.5 \cdot 10^{-1}$ & $5.4 \cdot 10^{-1}$ & $5.0 \cdot 10^{-1}$ \\
$500 \mathrm{GeV}$ & 7 & $7.9 \cdot 10^{-2}$ & $5.1 \cdot 10^{-2}$ & $8.4 \cdot 10^{-2}$ & $5.4 \cdot 10^{-2}$ & $7.6 \cdot 10^{-5}$ & $4.0 \cdot 10^{-1}$ & $3.7 \cdot 10^{-1}$ & $2.8 \cdot 10^{-1}$ \\
\hline
\end{tabular}

TABLE I: Cross sections (in fb) for the associated production of a charged Higgs with a hard jet: $p_{T, j}>100 \mathrm{GeV}$. The label 2HDM denotes a two-Higgs-doublet of type II, while MFV and SUSY refer to the complete set of supersymmetric diagrams, assuming MFV and beyond. The SUSY parameters are given in Eq.(14). Beyond MFV we choose $\delta_{L R, 31}^{u}=0.5$. The label $\left(m_{s}=0\right)$ means a zero strange Yukawa, $\left(m_{q}=0\right)$ indicates that all quark (except top) Yukawas are neglected. In this case only $D$-term couplings contribute within MFV.

other hand, $A_{3 i}^{u}$ and $m_{\tilde{U}_{R} i 3}^{2}$ are only very loosely bounded by flavor physics, the $L R$ chirality flip by Eq. (20). The reason is that these entries involve right-handed squarks $\tilde{u}_{R}$ and $\tilde{c}_{R}$; those enter FCNC processes with external down quarks only via higgsino vertices proportional to the small up and charm Yukawa. To circumvent this Yukawa suppression, we could combine $\tilde{t}-\tilde{u}_{L}\left(\tilde{c}_{L}\right)$ mixing with a subsequent generational-diagonal left-right mixing $\tilde{u}_{R}-\tilde{u}_{L}\left(\tilde{c}_{R}-\tilde{c}_{L}\right)$. However, generation-diagonal mixing is strongly constrained by the quark masses (18).

Further constraints on flavor mixing could arise from $B$-meson decays into $\tau \nu$ final states, which also receive contributions from a charged-Higgs exchange. $B$-factory experiments determine the $B_{u}^{-} \rightarrow \tau \bar{\nu}$ branching ratio to be in agreement with the Standard Model, within substantial theoretical and experimental uncertainties [47]. Since for our moderate values of $\tan \beta$ the $H^{ \pm}$-mediated amplitude cannot compete with the tree-level $W$ exchange, $B_{u}^{-} \rightarrow \tau \bar{\nu}$ data do not put additional constraints on the up squarks.

We have seen that $\delta_{L R 3 i}^{u}$ and $\delta_{R R i 3}^{u}(i=1,2)$ are currently the least constrained flavored SUSY couplings. Kaon, charm, and $B$-physics experiments are largely insensitive to the mixing of $\tilde{u}_{R}$ or $\tilde{c}_{R}$ with stops. The latter has impact on FCNC top decays, see also [49]. In this work we investigate the potential impact of these relevant $\delta_{3 i}^{u}$ on charged-Higgs collider searches.

We implement the constraints on the supersymmetric flavor sector into our code, and apply them at 90\% C.L.. Since we are interested in big effects only, we neglect flavor-diagonal SUSY contributions to the FCNCs. Recall that we are not in the large-tan $\beta$ region, where these corrections can be sizeable. Thus, we get complicated constraints in the higher-dimensional parameter space of the various $\delta$ s, which depend on squark and gaugino masses and wino-higgsino mixing. Note that all FCNC constraints vanish for mass-degenerate squarks because of the super-GIM mechanism and reappearance of flavor symmetry, respectively.

\section{CHARGED-HIGGS PRODUCTION WITH A HARD JET}

The generic chiral suppression that characterizes single-Higgs production and limits the cross section at tree level can be removed by adding an external gluon to the operator basis. Such operators can be of the form $i \bar{Q} \gamma_{\mu} Q H_{u} \stackrel{\leftrightarrow}{D^{\mu}} H_{u}^{C}$, leading to higher-dimensional $q \bar{q}^{\prime} H g$ operators after electroweak symmetry breaking. For a detailed discussion of the operator basis see e.g. Ref. [37]. It is of course by no means guaranteed that all possible operators are actually induced at the one-loop level in the MSSM. Some operators can be forbidden by symmetry.

To probe such operators at the LHC, we study charged-Higgs searches in association with a hard jet. Simple diagrams for this process can be derived from all single-Higgs production diagrams just radiating an additional gluon. The infrared divergences that occur for soft jets or jets that are collinear to the incoming partons are excluded by requiring a hard jet with transverse momentum $p_{T, j}>100 \mathrm{GeV}$.

Similar to single-Higgs production we are interested in supersymmetric loop corrections in and beyond MFV. Such diagrams are suppressed by $\alpha_{s}$, which means that when comparing them to tree-level rates in the twoHiggs-doublet model we should consistently compute the next-to-leading-order corrections to the tree-level. On the other hand, we know from single-Higgs production that the flavor effects we are interested in can be much larger than we expect next-to-leading-order QCD effects to be. Therefore, we ignore all gluonic nextto-leading order corrections to charged-Higgs production with a hard jet and limit our analysis to tree-level 

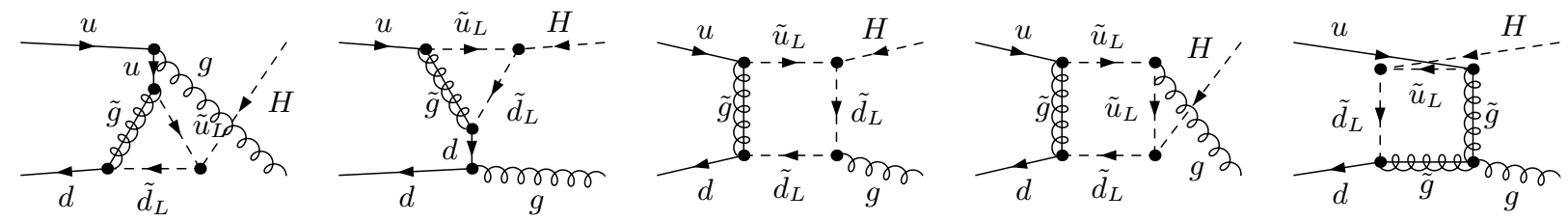

FIG. 5: SUSY QCD diagrams for $u \bar{d} \rightarrow g H^{ \pm}$with MFV and massless quarks.

rates in the two-Higgs-doublet model and additional supersymmetric one-loop corrections with and without the MFV assumption. Final-state top quarks introducing a top Yukawa we do not consider, because they lead to a completely different signature.

Unlike the amplitude for single-Higgs production, the amplitude for Higgs production with a hard jet does not vanish in the limit of zero quark masses, even in a two-Higgs-doublet model. There, contributions to non-chiral operators arise at two loops, when the charged Higgs couples to neutral Higgses and gauge bosons and not directly the fermions. However, such non-supersymmetric two-loop contributions have to be compared with the tree-level processes: modulo parton-density effects the bottom Yukawa competes with the weak coupling multiplied with two loop factors $\left(g^{2} /\left(16 \pi^{2}\right)\right)^{2} \sim 10^{-5}$, so we can safely neglect the two-loop non-chiral contributions as well.

In the first two columns of Table \ we list the hadronic tree-level cross sections for charged-Higgs-plus-jet production for a non-supersymmetric two-Higgs-doublet type-II model. Sharing this feature with the singleHiggs production discussed previously, the bottom Yukawa in the absence of a final-state top appears with a CKM suppression, leading to effective couplings of the order $m_{b} V_{c b} \sim m_{s} V_{c s}$. Parton densities will lightly enhance the strange-quark contribution compared to incoming bottoms. This numerical behavior is what we see in Table If at tree level the strange and the bottom Yukawas contribute at a comparable rate.

In the following analysis of charged-Higgs-plus-jet production in supersymmetry we also include Higgs decays. As long as the Higgs mass is small, $m_{H^{+}} \lesssim 200 \mathrm{GeV}$, the Higgs decay into a hadronic $\tau$ lepton is the most promising 10, 11]. For the lower-mass parameter point in Eq.(14) with its charged-Higgs mass of $188 \mathrm{GeV}$, we find $\operatorname{BR}\left(H^{-} \rightarrow \tau \bar{\nu}\right)=71 \%$, with a taggable hadronic $\tau$ branching ratio of around roughly two thirds [32]. The dominant background to this signature is clearly $W+$ jet production, again with the $W$ decaying to a hadronic $\tau$. For $p_{T, j}>100 \mathrm{GeV}$ the corresponding cross section is about $1 \mathrm{nb}$.

\section{A. MFV Loops and Decoupling}

The difference between the two-Higgs-doublet model and higher-dimensional operators realized by supersymmetric one-loop diagrams are additional Higgs couplings to squarks. We discuss those in Section [I] assuming MFV, $F$-term and $A$-term couplings of the Higgs to two squarks are proportional to the quark masses, which means that supersymmetric one-loop amplitudes are expected to be of the size of typical supersymmetric NLO corrections. In contrast, the $D$-term couplings shown in Eq.(7) are gauge couplings, which means they could be considerably larger than light-flavor Yukawas. This formal enhancement is a novel aspect of associated chargedHiggs production with a hard jet. For single-Higgs production, such $D$-term couplings do not contribute, because they are $L L$ diagonal in the squarks and do not introduce the necessary left-right mixing without additional beyond-MFV contributions.

Since it circumvents the Yukawa suppression of the amplitude in the two-Higgs-doublet model, one might expect the $D$-term contribution to charged-Higgs production with a jet to be significant. The corresponding gluino-squark diagrams are shown in Fig. 5. Chargino and neutralino loops are neglected due to their smaller gauge coupling. At the LHC, a mixed quark-gluon initial state yields the largest cross section for heavy-particle production, because it is a good compromise between the high- $x$ valence quark parton densities and the large gluon luminosity at lower $x$. 
The general amplitude for the partonic subprocess $u_{i}+\bar{d}_{j} \rightarrow H^{+}+g$ is given in terms of form factors as

$$
\mathcal{A}^{i j}=\sum_{\sigma} \sum_{k=1}^{6} \mathcal{F}_{k}^{i j, \sigma} \mathcal{M}_{k}^{i j, \sigma}, \quad \sigma=L, R
$$

with 12 standard matrix elements [50]

$$
\begin{array}{llrl}
\mathcal{M}_{1}^{i j, \sigma} & =\bar{v}_{j}\left(p_{2}\right) \notin P_{\sigma} u_{i}\left(p_{1}\right), & & \mathcal{M}_{4}^{i j, \sigma}=\bar{v}_{j}\left(p_{2}\right) \not k_{2} \notin P_{\sigma} u_{i}\left(p_{1}\right), \\
\mathcal{M}_{2}^{i j, \sigma}=\bar{v}_{j}\left(p_{2}\right) \not k_{2} P_{\sigma} u_{i}\left(p_{1}\right)\left(\varepsilon \cdot p_{1}\right), & & \mathcal{M}_{5}^{i j, \sigma}=\bar{v}_{j}\left(p_{2}\right) P_{\sigma} u_{i}\left(p_{1}\right)\left(\varepsilon \cdot p_{1}\right), \\
\mathcal{M}_{3}^{i j, \sigma}=\bar{v}_{j}\left(p_{2}\right) \not k_{2} P_{\sigma} u_{i}\left(p_{1}\right)\left(\varepsilon \cdot p_{2}\right), & & \mathcal{M}_{6}^{i j, \sigma}=\bar{v}_{j}\left(p_{2}\right) P_{\sigma} u_{i}\left(p_{1}\right)\left(\varepsilon \cdot p_{2}\right) .
\end{array}
$$

The momenta are assigned as $u_{i}\left(p_{1}\right), \bar{d}_{j}\left(p_{2}\right), H^{+}\left(k_{1}\right), g\left(k_{2}\right)$, the corresponding Mandelstam variables are $s=$ $\left(p_{1}+p_{2}\right)^{2}, t=\left(p_{1}-k_{1}\right)^{2}, u=\left(p_{1}-k_{2}\right)^{2}$, and $\varepsilon$ is the polarization vector of the gluon. $S U(3)$ gauge invariance implies a Ward identity, which means the amplitude has to vanish if we replace the external gluon polarization vector by the gluon momentum. This relates the different form factors to each other:

$$
\mathcal{F}_{1}^{i j, \sigma}+\mathcal{F}_{2}^{i j, \sigma}\left(p_{1} \cdot k_{2}\right)+\mathcal{F}_{3}^{i j, \sigma}\left(p_{2} \cdot k_{2}\right)=0, \quad \mathcal{F}_{5}^{i j, \sigma}\left(p_{1} \cdot k_{2}\right)+\mathcal{F}_{6}^{i j, \sigma}\left(p_{2} \cdot k_{2}\right)=0 .
$$

Numerical results for hadronic charged-Higgs-plus-jet production in MFV are presented in the second set of rows in Table I. We show the cross sections for the lower-mass parameter point (14), and vary $\tan \beta$ and $m_{H^{+}}$ as indicated. All supersymmetric loop diagrams share the usual loop-suppression factors. This means that in MFV the additional supersymmetric contributions are unlikely to numerically dominate over the tree-level rates in the two-Higgs-doublet model.

The purely $D$-term-induced contributions $\left(m_{q}=0\right)$ are numerically negligible, despite the fact that they avoid the chiral suppression. The reason is that the loop amplitude suffers from an additional mass suppression $1 / M_{\mathrm{SUSY}}^{4}$ in the limit $m_{H^{+}}^{2}, m_{W}^{2}, s,|t|,|u| \ll M_{\mathrm{SUSY}}^{2}$, where $M_{\mathrm{SUSY}}$ denotes a common squark and gluino mass. It is not easy to see this decoupling in the explicit analytical result for the form factors, which is given in the appendix. The decoupling can be understood by applying power-counting in $M_{\text {SUSY }}$ to the individual form factors in combination with the gauge-invariance relation (26). Naive power-counting suggests a scaling $\propto 1 / M_{\text {SUSY }}^{2}$ for the form factors, but including the Lorentz structure of the loop integrals reveals that only $\mathcal{F}_{1}^{i j, \sigma}$ can receive contributions of this order, while the other form factors scale $\propto 1 / M_{\text {SUSY }}^{4}$. Thus, Eq.(26) shows that all contributions in $\mathcal{F}_{1}^{i j, \sigma}$ proportional to $1 / M_{\text {SUSY }}^{2}$ have to cancel. We have explicitly verified this fact by performing a large-mass expansion [51] of the SUSY-QCD diagrams in the relevant SUSY masses, confirming that the one-loop amplitude with $D$-term couplings scales like

$$
\mathcal{A}_{\mathrm{D}-\mathrm{qerm}}^{\tilde{q} \tilde{g}} \propto \frac{g_{s}^{3} g}{M_{\mathrm{SUSY}}^{4}} \sin (2 \beta) .
$$

This means that the pure $D$-term contribution to the charged-Higgs plus a hard jet cross section decouples as $\sigma \propto 1 / M_{\text {SUSY }}^{8}$, four powers of $M_{\text {SUSY }}$ faster than the leading supersymmetric cross section (with finite quark masses or not imposing MFV).

Comparing the different Yukawas, Table 1 also shows that similarly to single-Higgs production and to the two-Higgs-doublet model the contribution of the strange Yukawa is non-negligible. To see the typical behavior of the MFV amplitudes we show the LHC cross section of a charged Higgs boson with a hard jet $\left(p_{T, j}>100 \mathrm{GeV}\right)$ as a function of $m_{H^{ \pm}}$and $\tan \beta$ in Fig. 6, with and without the branching ratio to hadronic $\tau$ 's. The upper panels show the contributions from $D$ terms only, while the lower panels include all supersymmetric MFV contributions. We start from the lower-mass parameter point (14). As expected, the rates drop dramatically for heavier Higgs masses, even worse once we include the Higgs decay. The $\tan \beta$ dependence still shows the original motivation to consider such loop-induced processes, and in particular the $D$ terms: for those, the rates are largest for small values of $\tan \beta$, where all other known searches fail. However, because of the unexpectedly large mass suppression, Yukawa couplings are numerically dominant, as indicated by the different scales on the $y$ axes in Fig. 6. Possible large supersymmetric corrections in this process can only occur beyond MFV — just like for single-Higgs production. 

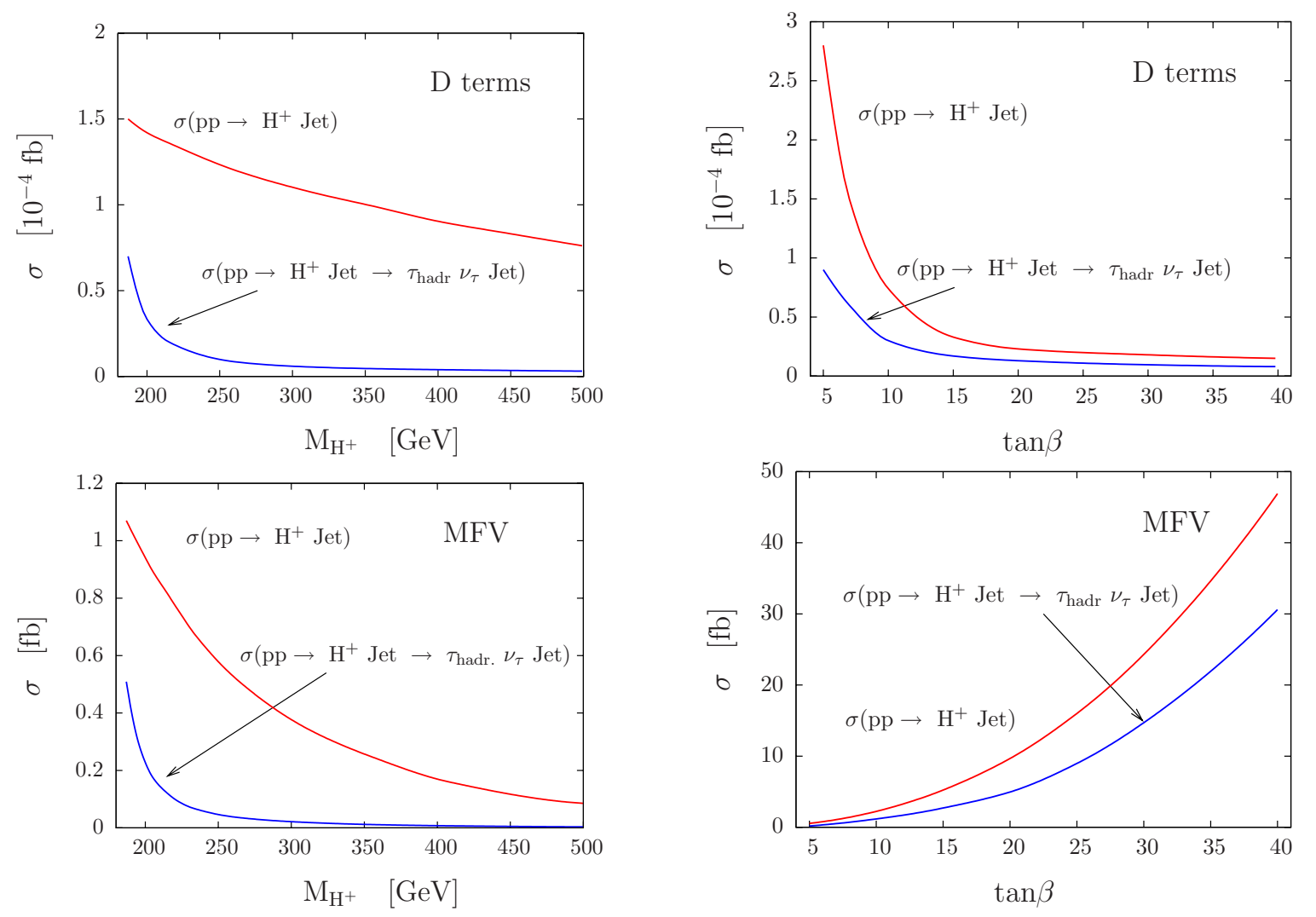

FIG. 6: Production rates for a charged Higgs with a hard jet including SUSY loops. Upper: assuming MFV with $D$-term contributions only. Lower: assuming MFV, but including all couplings. The supersymmetric parameters are listed in Eq.(14). We vary only the Higgs-sector parameters via the charged-Higgs mass and tan $\beta$. We include the Higgs decay into a hadronic $\tau$ plus $\nu_{\tau}$ (lower curves).

\section{B. Beyond MFV}

In contrast to single-Higgs production, the operator basis for Higgs-plus-jet production does not get significantly extended when we introduce beyond-MFV effects. However, just like for single-Higgs production the effective vertices shown in Fig. 1 will get significantly enhanced once we allow for sizeable $\delta_{A B, i j}^{u}$. Of course, to get a reliable account of the size of such effects we have to take into account the current limits on the flavor sector beyond MFV.

In this section we consider squark mixing between the first and third generation. The corresponding cross sections from second- and third-generation mixing are very similar, but slightly reduced due to the reduced charm parton density. The largely unconstrained $\delta_{R R, 13}^{u}$ and $\delta_{L R, 31}^{u}$ can have a sizeable effect on the chargedHiggs production rate. We already see this in the last set of columns in Tab. [I independent of the Yukawas, flavor effects beyond MFV can enhance the rate by a factor of five, compared to the tree-level process or compared to the MFV case. The same effect we see in the left panel of Fig. 7 where we show the variation of the Higgs cross section times branching ratio to a hadronic $\tau$ as a function of the $\delta^{u}$, each of them varied independently. For example, $\left|\delta_{L R, 31}^{u}\right|>0.2$ outgrows the tree-level results for the SUSY parameters listed in Eq.(14).

The bounds on the four considered $\delta^{u}$ mixings are different: The mass-matrix entries $\delta_{L L, 13}^{u}$ and $\delta_{L R, 13}^{u}$ are quite constrained. Their impact shown in Fig. 7 would not be allowed by flavor-physics constraints if only one of the $\delta$ 's was varied at a time. We nevertheless show the curves, because there might be cancellations induced by correlations between different deltas in the rare-decay observables. The four curves illustrate that the contribution of the different parameters beyond MFV are generically of similar size. To indicate how we would attempt to reduce the $W$ background we also show the distributions in the transverse mass

$$
m_{T, H}^{2}=\left(\left|\vec{p}_{T, \text { hadr }}\right|+\left|\vec{p}_{T, \text { miss }}\right|\right)^{2}-\left(\vec{p}_{T, \text { hadr }}+\vec{p}_{T, \text { miss }}\right)^{2}
$$



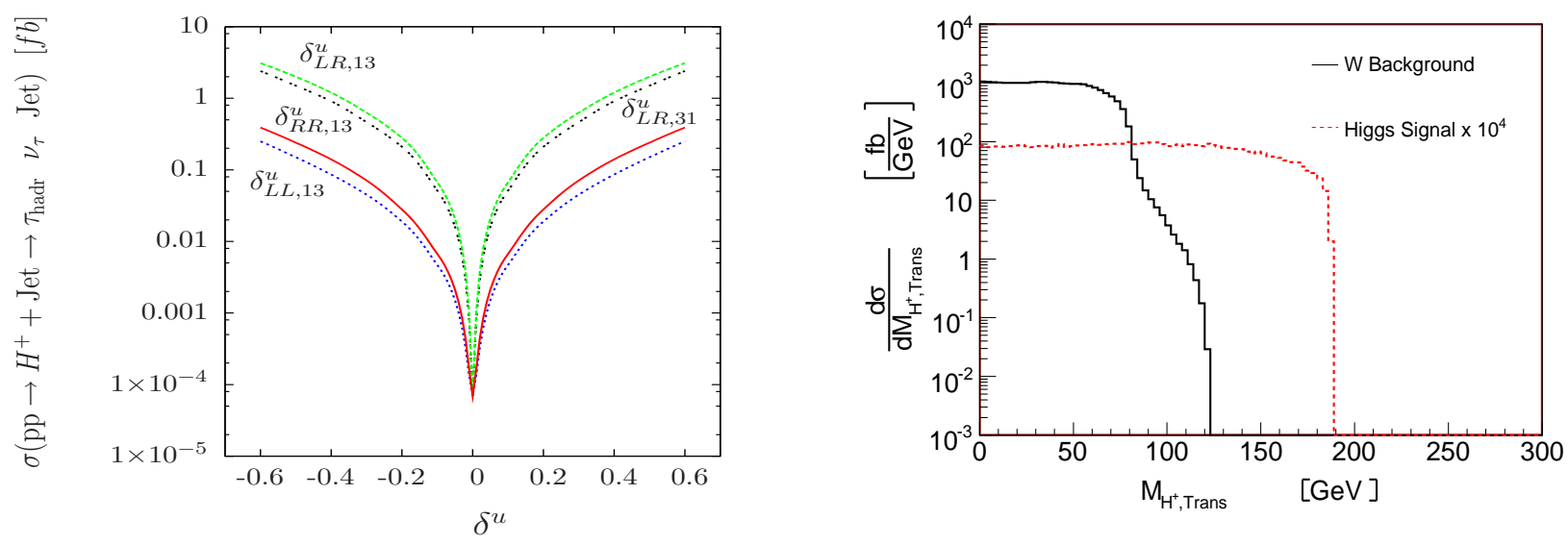

FIG. 7: Left: Cross sections for charged-Higgs production with a hard jet including the decay into a hadronic $\tau$ with beyond-MFV effects. The flavor-diagonal parameters correspond to the lower-mass scenario (14). We vary different $\delta_{A B, i j}^{u}$, one at a time, and assume $m_{q}=0$. Right: transverse-mass distributions for charged-Higgs production with a jet including a decay assuming beyond-MFV $\left(\delta_{L R, 31}^{u}=0.5\right)$. We also show the $W+$ jet background [52].

for the Higgs signal and for the $W$ background. For sufficiently large Higgs masses and modulo detectorresolution effect mostly on the missing transverse momentum vector, we could use such a distribution to enhance the signal over the background.

An interesting side aspect of Higgs-plus-jet production via different supersymmetric couplings can be seen in Fig. 8, in the left panel we show the $p_{T, j}$ distribution (equivalent to $p_{T, H}$ ) only taking into account $D$-term couplings in squark-gluino boxes and vertices. For small transverse momenta the cross section is finite, because the loops with $D$-term couplings have no counterpart in single-Higgs production and the $2 \rightarrow 2$ process is not an infrared-sensitive real-emission correction. Moreover, the heavy particles in the box define the typical energy scale of the process and show a threshold behavior around $p_{T} \sim 500 \mathrm{GeV}$. On the other hand, in the right panel we see that the $p_{T}$ distributions for the Higgs signal and the $W$ background look very similar. Both are infrared divergent for small values $p_{T}$. This infrared (soft and collinear) divergences will of course be canceled by virtual corrections and factorization contributions to the single-Higgs or single- $W$ processes. A proper description of the $p_{T}$ spectrum in the small- $p_{T}$ domain would require soft-gluon resummation.

In Figs. 7 and 8 we see how the Standard-Model background to charged-Higgs production is still overwhelming. On the other hand, the transverse-mass distribution also shows the background cut off above $m_{T}=m_{W}$. While detector effects will smear out this distribution, it might allow us to improve the signal-background ratio to a level where other cuts become useful. Probably, the transverse momentum of additional jets would be one of those signatures.

\section{OUTLOOK}

According to the current state of the art, charged-Higgs searches at the LHC have to rely on a tan $\beta$ enhanced bottom Yukawa for a sufficiently large production cross section. We studied two types of loop-induced production mechanisms which can significantly increase the production cross section for small Higgs masses and small values of $\tan \beta$ :

Single-charged Higgs production in $p p$ collisions in a general two-Higgs-doublet model is suppressed by either light-generation quark Yukawas or by small CKM mixing. For models with minimal flavor violation, this chiral suppression is generic and cannot be lifted by, e.g. , supersymmetric loops. If we allow for general squark mixing, additional loop-induced contributions arise. Here, the left-right chiral flip does not require a quark mass, but can proceed via squark mixing. We find that such contributions can enhance the single-charged-Higgs production cross section by almost an order of magnitude, even after including all current bounds on squark-flavor mixing.

Charged-Higgs production in association with a hard jet can be induced by supersymmetric $D$ terms. These are proportional to the weak gauge coupling and therefore appear in the one-loop amplitudes even in the limit 

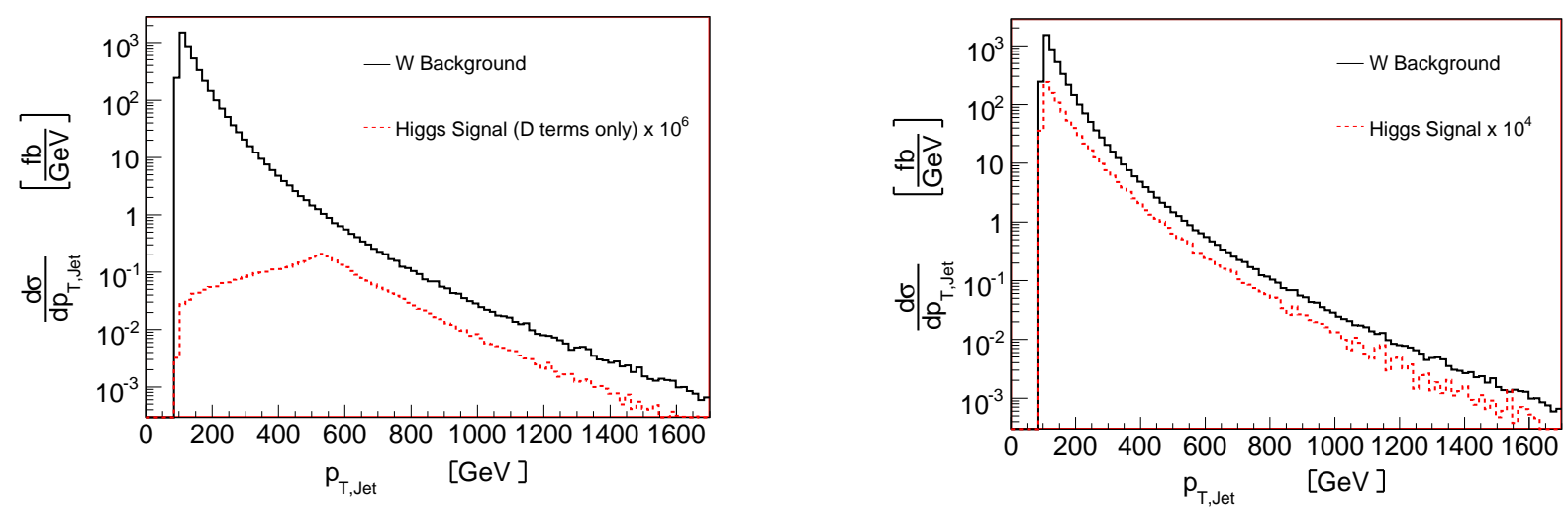

FIG. 8: Transverse-momentum distributions for charged-Higgs production with a jet including the decay to a hadronic $\tau$. We also show the scaled background distributions from $W+$ jet production [52]. The left panel shows MFV and $D$ terms only with $m_{q}=0$, the right panel includes beyond-MFV effects $\left(\delta_{L R, 31}^{u}=0.5\right)$. All other parameters given in Eq.(14).

of vanishing quark masses. We find, however, that although chirally not suppressed, the $D$-term contribution is only a small fraction of the supersymmetric amplitude, due to its faster decoupling with heavy superpartner masses. Just like in the single-charged Higgs case, only beyond-MFV contributions can enhance the associated charged-Higgs rate significantly above the two-Higgs-doublet model.

We find that the dominant source of genuine supersymmetric flavor enhancement in the charged-Higgs production rate is the soft-breaking $A$-term for up-type squarks, specifically $A_{3 i}^{u}$. It mixes the doublet-stop with light-generation singlets. This term is essentially unconstrained by flavor physics data, which are however sensitive to the chirality flipped term $A_{i 3}^{u}$. Based on theory prejudice these off-diagonal $A$-terms should be small [53],

$$
\begin{aligned}
\delta_{L R i j}^{q} & \sim \frac{m_{q_{i}} m_{q_{j}}}{\tilde{m}^{2}} \quad \text { (alignment) }, \\
\delta_{L R 3 j}^{u} \sim \frac{V_{j b}^{*} m_{u_{j}}}{\tilde{m}}, & \delta_{L R i 3}^{u} \sim \frac{V_{t i}^{*} m_{t}}{\tilde{m}} \text { (abelian flavor). }
\end{aligned}
$$

From Eq.(30) it follows further that squark mixing involving a doublet stop $\delta_{L R 3 j}^{u}$ is suppressed with respect to a singlet stop $\delta_{L R i 3}^{u}$ by a factor $m_{u_{j}} / m_{t}$.

We stress that the effects involving mixing of $\tilde{u}_{R}$ or $\tilde{c}_{R}$ with stops are unvisible to kaon, charm, and $B$ experiments. Hence, collider searches for enhanced charged-Higgs production cross sections probe a unique sector of flavor. A discovery would signal besides a breakdown of the Standard Model a quite non-standard solution to the flavor puzzle, including a breakdown of the minimal-flavor-violation hypothesis (see also [54] for MFV tests at the LHC).

At present, we cannot firmly claim that these flavor-induced charged-Higgs production rates at small $\tan \beta$ rates lead to observable signals over the large $W$-production backgrounds; we leave the conclusions to a detailed signal-background analysis, which carefully has to include detector effects.

\section{Acknowledgments}

GH is happy to thank Thorsten Feldmann for a stimulating discussion. We are particularly grateful to Michael Rauch for his help with FormCalc/HadCalc. MS and TP are grateful to the Max-Planck-Institute for Physics for their continuous hospitality. MS is grateful to Sebastian Jäger for helpful discussions. The work of GH is supported in part by Bundesministerium für Bildung und Forschung, Berlin-Bonn. This work is supported in part by the European Community's Marie-Curie Research Training Network HEPTOOLS under contract MRTN-CT-2006-035505. 


\section{Appendix}

In this appendix we give details about the super-CKM basis, the superpotential, supersymmetry breaking, and scalar mass matrices. Moreover, we give all numerical details in computing the $\overline{\mathrm{MS}}$ quark masses as well as explicit analytical results for the $D$-term-induced form factors for $\mathrm{H}^{+}$-plus-jet production.

\section{A. Super-CKM Basis}

Following the SUSY conventions of Ref. [55] except for $Y_{\text {here }}^{d}=-Y_{\text {Rosiek }}^{d}$ and $A_{\text {here }}^{u}=-A_{\text {Rosiek }}^{u}$ the MSSM superpotential is given as

$$
W=Q_{i} Y_{i j}^{u} U_{j} H_{u}-Q_{i} Y_{i j}^{d} D_{j} H_{d}+\mu H_{u} H_{d}
$$

where we make flavor indices $i, j=1,2,3$ explicit. The symbols $Q, U, D, H_{d}, H_{u}$ used for the superfields should not be confused with the same symbols used for the quark and Higgs fields in the main text. The superfields $Q, U, D$ and $H_{d}, H_{u}$ transform under $S U(3)_{C} \times S U(2)_{L} \times U(1)_{Y}$ as

$$
Q \equiv\left(3,2, \frac{1}{6}\right), \quad U \equiv\left(\overline{3}, 1,-\frac{2}{3}\right), \quad D \equiv\left(\overline{3}, 1, \frac{1}{3}\right), \quad H_{d} \equiv\left(1,2,-\frac{1}{2}\right), \quad H_{u} \equiv\left(1,2, \frac{1}{2}\right) .
$$

In the superpotential (A-1) and in the soft-breaking Lagrangian, see Eq. (A-6) below, we suppress $S U(2)$ contractions for all doublets, e.g. $H_{d} H_{u} \equiv \epsilon_{i j} H_{d i} H_{u j}$ with $\epsilon_{12}=+1$. (Note that $\epsilon_{12}^{\text {Rosiek }}=-1$.) The two Higgs doublets in the supersymmetric Lagrangian are defined in terms of their components $H_{d}=\left(H_{d}^{0}, H_{d}^{-}\right)^{T}$ and $H_{u}=\left(H_{u}^{+}, H_{u}^{0}\right)^{T}$. The scalar (fermionic) parts of the superfields $Q, U$, and $D$ are denoted as $\tilde{Q}\left(\Psi_{Q}\right), \tilde{U}^{*}\left(\Psi_{U}^{C}\right)$, and $\tilde{D}^{*}\left(\Psi_{D}^{C}\right)$ below, respectively, where $\Psi^{C}$ is the charge-conjugate of the fermion field $\Psi$.

Translating the three generations of flavor or weak eigenstates $Q, U, D$ with $Q=\left(U_{L}, D_{L}\right)^{T}$ (denoted by capital letters) into mass eigenstates $u_{L}, d_{L}, u_{R}, d_{R}$ and $\tilde{u}_{L}, \tilde{d}_{L}, \tilde{u}_{R}, \tilde{d}_{R}$ (denoted by small letters) defines the unitary transformations $V^{u, d}, U^{u, d}$,

$$
\begin{aligned}
u_{L} \equiv V^{u} \Psi_{U_{L}}, & d_{L} \equiv V^{d} \Psi_{D_{L}}, & u_{R} \equiv U^{u} \Psi_{U}, & d_{R} \equiv U^{d} \Psi_{D}, \\
\tilde{u}_{L}=V^{u} \tilde{U}_{L}, & \tilde{d}_{L}=V^{d} \tilde{D}_{L}, & \tilde{u}_{R}=U^{u} \tilde{U}, & \tilde{d}_{R}=U^{d} \tilde{D}
\end{aligned}
$$

such that the fermion mass matrices are diagonal:

$$
V^{u} Y^{u *} U^{u \dagger}=\operatorname{diag}\left(y_{u}, y_{c}, y_{t}\right)=\operatorname{diag}\left(\frac{m_{u}}{v_{u}}, \frac{m_{c}}{v_{u}}, \frac{m_{t}}{v_{u}}\right), \quad V^{d} Y^{d *} U^{d \dagger}=\operatorname{diag}\left(y_{d}, y_{s}, y_{b}\right)=\operatorname{diag}\left(\frac{m_{d}}{v_{d}}, \frac{m_{s}}{v_{d}}, \frac{m_{b}}{v_{d}}\right) .
$$

Note that the one-to-one map between Yukawas and masses receives corrections from non-holomorphic terms, relevant for down-type fermions at large $\tan \beta[18]$. The CKM matrix is given as $V \equiv V^{u} V^{d \dagger}$. It is parameterized according to the quark flavors it connects in the charged-current interaction,

$$
V=\left(\begin{array}{lll}
V_{u d} & V_{u s} & V_{u b} \\
V_{c d} & V_{c s} & V_{c b} \\
V_{t d} & V_{t s} & V_{t b}
\end{array}\right) .
$$

Given all these fields we can write the relevant flavored part of the soft-breaking Lagrangian in (gauge eigenstate) component fields with flavor indices $i, j=1, . .3$ :

$$
\mathcal{L}_{\text {soft }}=-\tilde{U}_{i}^{*} m_{\tilde{U} i j}^{2} \tilde{U}_{j}-\tilde{D}_{i}^{*} m_{\tilde{D} i j}^{2} \tilde{D}_{j}-\tilde{Q}_{i}^{\dagger} m_{\tilde{Q} i j}^{2} \tilde{Q}_{j}-\left[\tilde{Q}_{i} \bar{A}_{i j}^{u} \tilde{U}_{j}^{*} H_{u}-\tilde{Q}_{i} \bar{A}_{i j}^{d} \tilde{D}_{j}^{*} H_{d}+\text { h.c. }\right] .
$$

Diagonalizing the quark fields according to Eq. (A-3) leads to the quark mass basis. Simultaneous rotation of the squarks leads to $\mathcal{L}_{\text {soft }}$ in the super-CKM basis:

$$
\begin{aligned}
\mathcal{L}_{\text {soft }} & =-\tilde{u}_{R i}^{*} m_{\tilde{U}_{R} i j}^{2} \tilde{u}_{R j}-\tilde{d}_{R i}^{*} m_{\tilde{D}_{R} i j}^{2} \tilde{d}_{R j}-\tilde{u}_{L i}^{*} m_{\tilde{U}_{L} i j}^{2} \tilde{u}_{L j}-\tilde{d}_{L i}^{*} m_{\tilde{D}_{L} i j}^{2} \tilde{d}_{L j} \\
& -\left[\tilde{u}_{L i} A_{i j}^{u} \tilde{u}_{R j}^{*} H_{u}^{0}-\tilde{d}_{L i} V_{k i} A_{k j}^{u} \tilde{u}_{R j}^{*} H_{u}^{+}-\tilde{u}_{L i} V_{i k}^{*} A_{k j}^{d} \tilde{d}_{R j}^{*} H_{d}^{-}+\tilde{d}_{L i} A_{i j}^{d} \tilde{d}_{R j}^{*} H_{d}^{0}+\text { h.c. }\right],
\end{aligned}
$$

where for $q=u, d$

$$
A^{q}=V^{* q} \bar{A}^{q} U^{q T}, \quad m_{\tilde{U}_{R}}^{2}=U^{u} m_{\tilde{U}}^{2} U^{u \dagger}, \quad m_{\tilde{D}_{R}}^{2}=U^{d} m_{\tilde{D}}^{2} U^{d \dagger}, \quad m_{\tilde{U}_{L}}^{2}=V^{u} m_{\tilde{Q}}^{2} V^{u \dagger}, \quad m_{\tilde{D}_{L}}^{2}=V^{d} m_{\tilde{Q}}^{2} V^{d \dagger} .
$$




\section{B. Squark Masses}

The entries in the $6 \times 6$ squark mass matrices $\mathcal{M}_{q}^{2},(q=u, d)$ in Eq.(4) stem from soft-breaking $A$-terms in

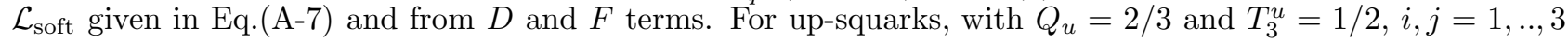
they read

$$
\begin{aligned}
& M_{u L L i j}^{2}=m_{\tilde{U}_{L} i j}^{2}+\left(m_{u_{i}}^{2}+\left(T_{3}^{u}-Q_{u} \sin ^{2} \theta_{w}\right) m_{Z}^{2} \cos 2 \beta\right) \delta_{i j}, \\
& M_{u R R i j}^{2}=m_{\tilde{U}_{R} i j}^{2}+\left(m_{u_{i}}^{2}+Q_{u} \sin ^{2} \theta_{w} m_{Z}^{2} \cos 2 \beta\right) \delta_{i j}, \\
& M_{u L R i j}^{2}=\left\langle H_{u}^{0}\right\rangle A_{i j}^{u}-m_{u_{i}} \mu \cot \beta \delta_{i j}
\end{aligned}
$$

while for down-squarks they read with $Q_{d}=-1 / 3$ and $T_{3}^{d}=-1 / 2$

$$
\begin{aligned}
& M_{d L L i j}^{2}=m_{\tilde{D}_{L} i j}^{2}+\left(m_{d_{i}}^{2}+\left(T_{3}^{d}-Q_{d} \sin ^{2} \theta_{w}\right) m_{Z}^{2} \cos 2 \beta\right) \delta_{i j}, \\
& M_{d R R i j}^{2}=m_{\tilde{D}_{R} i j}^{2}+\left(m_{d_{i}}^{2}+Q_{d} \sin ^{2} \theta_{w} m_{Z}^{2} \cos 2 \beta\right) \delta_{i j}, \\
& M_{d L R i j}^{2}=\left\langle H_{d}^{0}\right\rangle A_{i j}^{d}-m_{d_{i}} \mu \tan \beta \delta_{i j} .
\end{aligned}
$$

We recall that throughout this paper the SUSY-breaking parameters and the $\mu$-term are real quantities.

The full squark mass matrices $\mathcal{M}_{q}^{2}$ can be diagonalized with unitary transformations $Z^{q}$ to obtain the squark mass eigenstates $\tilde{q}_{1 i}, \tilde{q}_{2 i}$ :

$$
Z^{q} \mathcal{M}_{q}^{2} Z^{q \dagger}=\operatorname{diag}\left(m_{\widetilde{q}_{1 i}}^{2}, m_{\widetilde{q}_{2 i}}^{2}\right), \quad Z^{u}\left(\begin{array}{c}
\widetilde{u}_{L} \\
\widetilde{c}_{L} \\
\widetilde{t}_{L} \\
\widetilde{u}_{R} \\
\widetilde{c}_{R} \\
\widetilde{t}_{R}
\end{array}\right)=\left(\begin{array}{c}
\widetilde{u}_{1} \\
\widetilde{u}_{2} \\
\widetilde{u}_{3} \\
\widetilde{u}_{4} \\
\widetilde{u}_{5} \\
\widetilde{u}_{6}
\end{array}\right), \quad Z^{d}\left(\begin{array}{c}
\widetilde{d}_{L} \\
\widetilde{s}_{L} \\
\widetilde{b}_{L} \\
\widetilde{d}_{R} \\
\widetilde{s}_{R} \\
\widetilde{b}_{R}
\end{array}\right)=\left(\begin{array}{c}
\widetilde{d}_{1} \\
\widetilde{d}_{2} \\
\widetilde{d}_{3} \\
\widetilde{d}_{4} \\
\widetilde{d}_{5} \\
\widetilde{d}_{6}
\end{array}\right) .
$$

\section{Quark Masses}

We use the running quark masses at next-to-leading order

$$
m(\mu)=m\left(\mu_{0}\right)\left[\frac{\alpha_{s}(\mu)}{\alpha_{s}\left(\mu_{0}\right)}\right]^{\gamma_{m}^{(0)} /\left(2 \beta_{0}\right)}\left[1+\left(\frac{\gamma_{m}^{(1)}}{2 \beta_{0}}-\frac{\beta_{1} \gamma_{m}^{(0)}}{2 \beta_{0}^{2}}\right) \frac{\alpha_{s}(\mu)-\alpha_{s}\left(\mu_{0}\right)}{4 \pi}\right]
$$

Here, $\beta_{0}=11-2 / 3 N_{f}, \beta_{1}=102-38 / 3 N_{f}, \gamma_{m}^{(0)}=6 C_{F}$ and $\gamma_{m}^{(1)}=C_{F}\left(3 C_{F}+97-10 / 3 N_{f}\right)$, where $N_{f}$ denotes the number of quarks with $m_{f} \leq \mu$.

For our numerical analysis we use the numerical values for the quark masses [56]:

\begin{tabular}{|c|c|c|}
\hline$m_{u}(2 \mathrm{GeV})$ & $m_{d}(2 \mathrm{GeV})$ & $m_{s}(2 \mathrm{GeV})$ \\
$2.8 \pm 0.6 \mathrm{MeV}$ & $5.0 \pm 1.0 \mathrm{MeV}$ & $95 \pm 15 \mathrm{MeV}$ \\
\hline$m_{c}\left(m_{c}\right)$ & $m_{b}\left(m_{b}\right)$ & $m_{t}\left(m_{t}\right)$ \\
$1.28 \pm 0.05 \mathrm{GeV}$ & $4.22 \pm 0.05 \mathrm{GeV}$ & $163 \pm 3 \mathrm{GeV}$ \\
\hline$m_{u}\left(m_{Z}\right)$ & $m_{d}\left(m_{Z}\right)$ & $m_{s}\left(m_{Z}\right)$ \\
$1.7 \pm 0.4 \mathrm{MeV}$ & $3.0 \pm 0.6 \mathrm{MeV}$ & $54 \pm 8 \mathrm{MeV}$ \\
\hline$m_{c}\left(m_{Z}\right)$ & $m_{b}\left(m_{Z}\right)$ & $m_{t}\left(m_{Z}\right)$ \\
$0.62 \pm 0.03 \mathrm{GeV}$ & $2.87 \pm 0.03 \mathrm{GeV}$ & $171 \pm 3 \mathrm{GeV}$ \\
\hline
\end{tabular}

\section{Form factors for $D$-term contributions to $H^{+}$-plus-jet production}

Here we present explicit results for the form factors for $H^{+}$-plus-jet production induced by supersymmetric $D$ terms assuming MFV. For massless light quarks the form factors $\mathcal{F}_{4,5,6}^{i j, \sigma}$ vanish. For massless quarks we also 
see $\mathcal{F}_{1 \ldots 6}^{i j, R}=0$, since $D$ terms couple the $H^{ \pm}$only to the left-handed squarks $(\sigma=L)$. Following Eq. (26) only two out of the remaining form factors are independent. Choosing $\mathcal{F}_{1,2}^{i j, L}$, the whole result can be expressed in terms of

$$
\begin{aligned}
\mathcal{F}_{1}^{i j, L}=e g_{s}^{3} \frac{m_{W}}{\pi^{2}} \frac{V_{i j}^{*} \sin 2 \beta}{12 \sqrt{2} \sin \theta_{w}}[ & -C_{1}\left(c_{1}\right)-C_{1}\left(c_{2}\right)+\frac{D_{00}\left(d_{1}\right)}{4}+\frac{D_{00}\left(d_{2}\right)}{4} \\
+\frac{9}{8}[ & D_{0}\left(d_{3}\right) m_{\tilde{g}}^{2}-D_{1}\left(d_{3}\right) m_{H^{+}}^{2}-D_{2}\left(d_{3}\right) u-2 D_{00}\left(d_{3}\right)-D_{11}\left(d_{3}\right) m_{H^{+}}^{2} \\
& \left.\left.\quad-D_{12}\left(d_{3}\right)\left(m_{H^{+}}^{2}+u\right)-D_{13}\left(d_{3}\right)(s+u)-D_{22}\left(d_{3}\right) u-D_{23}\left(d_{3}\right) u\right]\right], \\
\mathcal{F}_{2}^{i j, L}=e g_{s}^{3} \frac{m_{W}}{\pi^{2}} \frac{V_{i j}^{*} \sin 2 \beta}{48 \sqrt{2} \sin \theta_{w}}\left[\begin{array}{l}
D_{23}\left(d_{1}\right)-D_{2}\left(d_{2}\right)-D_{22}\left(d_{2}\right)-D_{23}\left(d_{2}\right) \\
-9
\end{array}\right. & {\left.\left[D_{1}\left(d_{3}\right)+D_{2}\left(d_{3}\right)+D_{11}\left(d_{3}\right)+2 D_{12}\left(d_{3}\right)+D_{13}\left(d_{3}\right)+D_{22}\left(d_{3}\right)+D_{23}\left(d_{3}\right)\right]\right], }
\end{aligned}
$$

where the tensor coefficients $C_{i \ldots}, D_{i \ldots}$ are defined as in Ref. [57]. We use the following abbreviations to specify the arguments of the three-point and four-point integrals:

$$
\begin{aligned}
& c_{1}=\left(m_{H^{+}}^{2}, t, 0, m_{\tilde{u}_{j}}, m_{\tilde{d}_{i}}, m_{\tilde{g}}\right), \\
& c_{2}=\left(m_{H^{+}}^{2}, u, 0, m_{\tilde{d}_{j}}, m_{\tilde{u}_{i}}, m_{\tilde{g}}\right), \\
& d_{1}=\left(0,0, m_{H^{+}}^{2}, 0, s, t, m_{\tilde{g}}, m_{\tilde{d}_{j}}, m_{\tilde{d}_{j}}, m_{\tilde{u}_{i}}\right), \\
& d_{2}=\left(0, m_{H^{+}}^{2}, 0,0, s, u, m_{\tilde{g}}, m_{\tilde{d}_{j}}, m_{\tilde{u}_{i}}, m_{\tilde{u}_{i}}\right), \\
& d_{3}=\left(m_{H^{+}}^{2}, 0,0,0, t, u, m_{\tilde{u}_{i}}, m_{\tilde{d}_{j}}, m_{\tilde{g}}, m_{\tilde{g}}\right),
\end{aligned}
$$

They are connected to the ordering scheme for the arguments of the loop functions defined in Ref. [57] as

$$
\begin{aligned}
& c=\left(p_{1}^{2},\left(p_{1}-p_{2}\right)^{2}, p_{2}^{2}, m_{1}, m_{2}, m_{3}\right) \equiv\left(p_{1}, p_{2}, m_{1}, m_{2}, m_{3}\right), \\
& d=\left(p_{1}^{2},\left(p_{1}-p_{2}\right)^{2},\left(p_{2}-p_{3}\right)^{2}, p_{3}^{2},\left(p_{1}-p_{3}\right)^{2}, p_{2}^{2}, m_{1}, m_{2}, m_{3}, m_{4}\right) \equiv\left(p_{1}, p_{2}, p_{3}, m_{1}, m_{2}, m_{3}, m_{4}\right) .
\end{aligned}
$$

[1] See e.g. : J. F. Gunion, H. E. Haber, G. L. Kane and S. Dawson, SCIPP-89/13; A. Djouadi, arXiv:hep-ph/0503173

[2] For the most recent results see: http://lepewwg.web.cern.ch

[3] V. Büscher and K. Jakobs, Int. J. Mod. Phys. A 20, 2523 (2005); T. Plehn, D. Rainwater and D. Zeppenfeld, Phys. Lett. B 454, 297 (1999); T. Plehn, D. Rainwater and D. Zeppenfeld, Phys. Rev. D 61, 093005 (2000); M. Schumacher, arXiv:hep-ph/0410112.

[4] M. Dührssen, S. Heinemeyer, H. Logan, D. Rainwater, G. Weiglein and D. Zeppenfeld, Phys. Rev. D 70, 113009 $(2004)$

[5] R. M. Barnett, H. E. Haber and D. E. Soper, Nucl. Phys. B 306, 697 (1988); A. C. Bawa, C. S. Kim and A. D. Martin, Z. Phys. C 47, 75 (1990); V. D. Barger, R. J. Phillips and D. P. Roy, Phys. Lett. B 324, 236 (1994); J. L. Diaz-Cruz and O. A. Sampayo, Phys. Rev. D 50, 6820 (1994); S. Moretti and K. Odagiri, Phys. Rev. D 55, 5627 (1997).

[6] S. H. Zhu, Phys. Rev. D 67, 075006 (2003); J. Alwall and J. Rathsman, JHEP 0412, 050 (2004); N. Kidonakis, JHEP 0505, 011 (2005).

[7] T. Plehn, Phys. Rev. D 67, 014018 (2003).

[8] E. L. Berger, T. Han, J. Jiang and T. Plehn, Phys. Rev. D 71, 115012 (2005).

[9] J. C. Collins and W. K. Tung, Nucl. Phys. B 278, 934 (1986); M. A. G. Aivazis, J. C. Collins, F. I. Olness and W. K. Tung, Phys. Rev. D 50, 3102 (1994); F. I. Olness and W. K. Tung, Nucl. Phys. B 308, 813 (1988); M. Krämer, F. I. Olness and D. E. Soper, Phys. Rev. D 62, 096007 (2000); E. Boos and T. Plehn, Phys. Rev. D 69, 094005 (2004). 
[10] D. P. Roy, Phys. Lett. B 459, 607 (1999).

[11] K. A. Assamagan and Y. Coadou, Acta Phys. Polon. B 33, 707 (2002); Y. Coadou, FERMILAB-THESIS-2003-31; R. Kinnunen and A. Nikitenko, report CMS note 2003/006.

[12] J. F. Gunion, Phys. Lett. B 322, 125 (1994); J. A. Coarasa, D. Garcia, J. Guasch, R. A. Jimenez and J. Sola, Phys. Lett. B 425, 329 (1998); S. Moretti and D. P. Roy, Phys. Lett. B 470, 209 (1999).

[13] K. A. Assamagan, Y. Coadou and A. Deandrea, report ATL-COM-PHYS-2002-002, arXiv:hep-ph/0203121, K. A. Assamagan and N. Gollub, Eur. Phys. J. C 39S2, 25 (2005); P. Salmi, R. Kinnunen and N. Stepanov, arXiv:hep-ph/0301166. S. Lowette, J. D'Hondt and P. Vanlaer, CERN-CMS-NOTE-2006-109; also see: S. Lowette, Ph.D thesis http://web.iihe.ac.be/ slowette

[14] F. Borzumati, J. L. Kneur and N. Polonsky, Phys. Rev. D 60, 115011 (1999). for the analysis results see: CMS TDR, Volume II, CERN/LHCC 2006-021, p.386.

[15] T. Plehn, M. Spira and P. M. Zerwas, Nucl. Phys. B 479, 46 (1996) [Erratum-ibid. B 531, 655 (1998)]; S. Dawson, S. Dittmaier and M. Spira, Phys. Rev. D 58, 115012 (1998); A. Djouadi, W. Kilian, M. Mühlleitner and P. M. Zerwas, Eur. Phys. J. C 10, 45 (1999); A. A. Barrientos Bendezu and B. A. Kniehl, Phys. Rev. D 64, 035006 (2001); U. Baur, T. Plehn and D. L. Rainwater, Phys. Rev. D 69, 053004 (2004).

[16] D. A. Dicus, J. L. Hewett, C. Kao and T. G. Rizzo, Phys. Rev. D 40, 787 (1989); A. A. Barrientos Bendezu and B. A. Kniehl, Phys. Rev. D 59, 015009 (1998); and Phys. Rev. D 63, 015009 (2001); O. Brein, W. Hollik and S. Kanemura, Phys. Rev. D 63, 095001 (2001); Z. Fei, M. Wen-Gan, J. Yi, H. Liang and W. Lang-Hui, Phys. Rev. D 63, 015002 (2000); W. Hollik and S. H. Zhu, Phys. Rev. D 65, 075015 (2002).

[17] J. F. Gunion, H. E. Haber, F. E. Paige, W. K. Tung and S. S. Willenbrock, Nucl. Phys. B 294, 621 (1987); S. S. D. Willenbrock, Phys. Rev. D 35, 173 (1987); A. Krause, T. Plehn, M. Spira and P. M. Zerwas, Nucl. Phys. B 519, 85 (1998); A. A. Barrientos Bendezu and B. A. Kniehl, Nucl. Phys. B 568, 305 (2000); O. Brein and W. Hollik, Eur. Phys. J. C 13, 175 (2000); E. Eichten, I. Hinchliffe, K. D. Lane and C. Quigg, Rev. Mod. Phys. 56, 579 (1984) [Addendum-ibid. 58, 1065 (1986)]; N. G. Deshpande, X. Tata and D. A. Dicus, Phys. Rev. D 29, 1527 (1984); for a recent update and overview, see e.g. : A. Alves and T. Plehn, Phys. Rev. D 71, 115014 (2005).

[18] L. J. Hall, R. Rattazzi and U. Sarid, Phys. Rev. D 50, 7048 (1994); M. Carena, M. Olechowski, S. Pokorski and C. E. Wagner, Nucl. Phys. B 426, 269 (1994); M. Carena, D. Garcia, U. Nierste and C. E. Wagner, Nucl. Phys. B 577, 88 (2000); A. Belyaev, D. Garcia, J. Guasch and J. Sola, Phys. Rev. D 65, 031701(R) (2002); J. Guasch, P. Häfliger and M. Spira, Phys. Rev. D 68, 115001 (2003).

[19] R. S. Chivukula and H. Georgi, Phys. Lett. B 188, 99 (1987); G. D'Ambrosio, G. F. Giudice, G. Isidori and A. Strumia, Nucl. Phys. B 645, 155 (2002).

[20] G. Hiller and M. Schmaltz, Phys. Rev. D 65, 096009 (2002).

[21] W. Altmannshofer, A. J. Buras and D. Guadagnoli, arXiv:hep-ph/0703200

[22] L. J. Hall, V. A. Kostelecky and S. Raby, Nucl. Phys. B 267, 415 (1986).

[23] J. S. Hagelin, S. Kelley and T. Tanaka, Nucl. Phys. B 415, 293 (1994); F. Gabbiani, E. Gabrielli, A. Masiero and L. Silvestrini, Nucl. Phys. B 477, 321 (1996); M. Misiak, S. Pokorski and J. Rosiek, Adv. Ser. Direct. High Energy Phys. 15, 795 (1998).

[24] J. A. Casas and S. Dimopoulos, Phys. Lett. B 387, 107 (1996).

[25] See, e.g. : Y. Nir, arXiv:hep-ph/0703235, and references therein.

[26] B. Aubert et al. [BABAR Collaboration], Phys. Rev. D 72, 052004 (2005); P. Koppenburg et al. [Belle Collaboration], Phys. Rev. Lett. 93, 061803 (2004); S. Chen et al. [CLEO Collaboration], Phys. Rev. Lett. 87, 251807 (2001); W.-M. Yao et al., J. Phys. Lett. G 33, 1 (2006); R. Barate et al. [ALEPH Collaboration], Phys. Lett. B 429, 169 (1998); H. F. A. Group(HFAG), arXiv:hep-ex/0505100

[27] P. L. Cho, M. Misiak and D. Wyler, Phys. Rev. D 54, 3329 (1996); J. L. Hewett and J. D. Wells, Phys. Rev. D 55, 5549 (1997); F. M. Borzumati and C. Greub, Phys. Rev. D 58, 074004 (1998) and Phys. Rev. D 59, 057501 (1999); G. Hiller and F. Krüger, Phys. Rev. D 69, 074020 (2004).

[28] K. Abe et al., Phys. Rev. Lett. 96, 221601 (2006); B. Aubert et al. [BABAR Collaboration], arXiv:hep-ex/0612017,

[29] B. Aubert et al. [BABAR Collaboration], Phys. Rev. Lett. 93, 081802 (2004). K. Abe et al. [Belle Collaboration], arXiv:hep-ex/0408119, A. Ali, E. Lunghi, C. Greub and G. Hiller, Phys. Rev. D 66, 034002 (2002); B. Grinstein, M. J. Savage and M. B. Wise, Nucl. Phys. B 319, 271 (1989); D. Guetta and E. Nardi, Phys. Rev. D 58, 012001 (1998).

[30] E. Lunghi, A. Masiero, I. Scimemi and L. Silvestrini, Nucl. Phys. B 568, 120 (2000); G. Buchalla, G. Hiller and G. Isidori, Phys. Rev. D 63, 014015 (2001); G. Hiller, Phys. Rev. D 66, 071502 (2002).

[31] [BABAR Collaboration], arXiv:hep-ex/0703018, A. Ali, P. Ball, L. T. Handoko and G. Hiller, Phys. Rev. D 61, 074024 (2000); P. Ball and R. Zwicky, Phys. Rev. D 71, 014015 (2005).

[32] W. M. Yao et al. [Particle Data Group], J. Phys. G 33 (2006) 1; E. Barberio et al. [Heavy Flavor Averaging Group (HFAG)], arXiv:hep-ex/0603003 http://www.slac.stanford.edu/xorg/hfag

[33] V. M. Abazov et al. [D0 Collaboration], Phys. Rev. Lett. 97, 021802 (2006); A. Abulencia et al. [CDF Collaboration], Phys. Rev. Lett. 97, 242003 (2006).

[34] S. Bertolini, F. Borzumati, A. Masiero and G. Ridolfi, Nucl. Phys. B 353, 591 (1991); G. C. Branco, G. C. Cho, Y. Kizukuri and N. Oshimo, Phys. Lett. B 337, 316 (1994).

[35] D. Becirevic et al., Nucl. Phys. B 634, 105 (2002); A. Bartl, T. Gajdosik, E. Lunghi, A. Masiero, W. Porod, H. Stremnitzer and O. Vives, Phys. Rev. D 64, 076009 (2001);

P. Ball, S. Khalil and E. Kou, Phys. Rev. D 69, 115011 (2004); M. Blanke, A. J. Buras, D. Guadagnoli and 
C. Tarantino, JHEP 0610, 003 (2006); T. Goto, T. Nihei and Y. Okada, Phys. Rev. D 53, 5233 (1996) [Erratumibid. D 54, 5904 (1996)].

[36] J. L. Diaz-Cruz, H. J. He and C. P. Yuan, Phys. Lett. B 530, 179 (2002); H. J. He and C. P. Yuan, Phys. Rev. Lett. 83, 28 (1999); C. Balazs, H. J. He and C. P. Yuan, Phys. Rev. D 60, 114001 (1999); S. R. Slabospitsky, arXiv:hep-ph/0203094.

[37] C. N. Leung, S. T. Love and S. Rao, Z. Phys. C 31, 433 (1986); W. Buchmüller and D. Wyler, Nucl. Phys. B 268, 621 (1986).

[38] S. P. Martin and M. T. Vaughn, Phys. Rev. D 50, 2282 (1994).

[39] G. Colangelo and G. Isidori, JHEP 9809, 009 (1998).

[40] T. Hahn, Comput. Phys. Commun. 140 (2001) 418; T. Hahn and C. Schappacher, Comput. Phys. Commun. 143 (2002) 54.

[41] T. Hahn and M. Perez-Victoria, Comput. Phys. Commun. 118 (1999) 153.

[42] T. Hahn and M. Rauch, Nucl. Phys. Proc. Suppl. 157, 236 (2006).

[43] J. Pumplin, D. R. Stump, J. Huston, H. L. Lai, P. Nadolsky and W. K. Tung, JHEP 0207 (2002) 012.

[44] For the most recent limits see: http://www-cdf.fnal.gov/physics/exotic/exotic.html; V. M. Abazov et al. [D0 Collaboration], Phys. Lett. B 638, 119 (2006).

[45] D. M. Pierce, J. A. Bagger, K. T. Matchev and R. j. Zhang, Nucl. Phys. B 491, 3 (1997).

[46] H. E. Haber, R. Hempfling and A. H. Hoang, Z. Phys. C 75, 539 (1997); M. Frank, T. Hahn, S. Heinemeyer, W. Hollik, H. Rzehak and G. Weiglein, arXiv:hep-ph/0611326

[47] K. Ikado et al., Phys. Rev. Lett. 97, 251802 (2006); B. Aubert et al. [BABAR Collaboration]; arXiv:hep-ex/0608019. A. G. Akeroyd and S. Recksiegel, J. Phys. G 29, 2311 (2003).

[48] S. Heinemeyer, W. Hollik, F. Merz and S. Penaranda, Eur. Phys. J. C 37, 481 (2004).

[49] J. Cao, G. Eilam, K. i. Hikasa and J. M. Yang, Phys. Rev. D 74, 031701 (2006); J. J. Cao, G. Eilam, M. Frank, K. Hikasa, G. L. Liu, I. Turan and J. M. Yang, Phys. Rev. D 75, 075021 (2007).

[50] A. Denner, Fortsch. Phys. 41, 307 (1993).

[51] V. A. Smirnov, Phys. Lett. B 394 (1997) 205.

[52] T. Stelzer and W. F. Long, Comput. Phys. Commun. 81, 357 (1994); F. Maltoni and T. Stelzer, JHEP 0302, 027 (2003); http://madgraph.phys.ucl.ac.be

[53] Y. Nir and N. Seiberg, Phys. Lett. B 309, 337 (1993).

[54] Y. Grossman, Y. Nir, J. Thaler, T. Volansky and J. Zupan, arXiv:0706.1845 [hep-ph].

[55] J. Rosiek, arXiv:hep-ph/9511250.

[56] See e.g. : M. Jamin, talk given at the University of Granada, March 2006.

[57] A. Denner and S. Dittmaier, Nucl. Phys. B 734, 62 (2006). 